\title{
A brute-force spectral approach for wave estimation using measured vessel motions
}

\author{
Ulrik D. Nielsen ${ }^{\mathrm{a}, \mathrm{b}}$, Astrid H. Brodtkorb ${ }^{\mathrm{b}}$, Asgeir J. Sørensen ${ }^{\mathrm{b}}$ \\ ${ }^{a}$ DTU Mechanical Engineering, Technical University of Denmark, DK-2800 Kgs. Lyngby, Denmark \\ ${ }^{b}$ Centre for Autonomous Marine Operations, AMOS-NTNU, NO-7491 Trondheim, Norway
}

\begin{abstract}
The article introduces a spectral procedure for sea state estimation based on measurements of motion responses of a ship in a short-crested seaway. The procedure relies fundamentally on the wave buoy analogy, but the wave spectrum estimate is obtained in a direct - brute-force - approach, and the procedure is simple in its mathematical formulation. The actual formulation is extending another recent work by including vessel advance speed and short-crested seas. Due to its simplicity, the procedure is computationally efficient, providing wave spectrum estimates in the order of a few seconds, and the estimation procedure will therefore be appealing to applications related to realtime, onboard control and decision support systems for safe and efficient marine operations. The procedure's performance is evaluated by use of numerical simulation of motion measurements, and it is shown that accurate wave spectrum estimates can be obtained for all wave directions in short-crested waves, taking the wave system to be composed by both wind generated sea and swell. Furthermore, the procedure is tested using full-scale motion data obtained from sea trials. Good wave estimations are achieved as compared to corresponding results from a free-floating (classical) wave buoy.
\end{abstract}

Keywords:

Wave spectrum estimation, wave-induced vessel responses, wave buoy analogy, Doppler Shift, spectrum transformation, sea trials data

Email address: udn@mek.dtu.dk (Ulrik D. Nielsen) 


\section{Introduction}

The level of autonomy within marine operations is increasing, and this trend is expected to continue in the future $[1,2,3]$. One area of autonomy is related to risk and performance evaluation of the actual operation, where focus may be on, say, cargo and passenger safety on a ship advancing in a seaway, hull girder integrity, fuel performance of the operating marine vessel, exact positioning and deterministic motion prediction of an offshore installation craft, etc. However, safety and fuel efficiency in open seas are compromised primarily because of the existence of ocean waves. Consequently, it is fundamentally important to have estimates of the wave system, the sea state, in which the marine craft or structure operates; otherwise, performance evaluation on safety and efficiency cannot be made.

One means to rely on for obtaining the sea state at a vessel's exact geographic position, in realtime, is that of the wave buoy analogy, where onboard sensor measurements of wave-induced (motion) responses are processed to yield the wave energy distribution of the encountered wave system $[4,5,6,7,8,9]$. In principle, any global wave-induced response can be used as input, although most studies on the wave buoy analogy are focused on motion components, for instance, heave, roll, and pitch. However, it is possible also to use, e.g., the wave-induced (vertical) bending moment amidships as input [10] if measurements and the corresponding transfer function are available. Various mathematical formulations of the wave buoy analogy exist and an overview of available procedures has been given by Nielsen [11]. Just recently, a new implementation of the wave buoy analogy has been suggested by Brodtkorb et al. [12]. The particular work considers dynamically positioned ships, without forward speed, and provides sea state estimation in long-crested waves.

The present study is a continuation of [12], with the aim to generalise the implementation to include measurements from a ship with advance speed, and letting the seaway be represented by short-crested (directional) waves. Two characteristics of the implementation are noteworthy: (1) the implementation is a brute-force spectral approach that allows for high computational efficiency, (2) the given solution applies (initially) to the encounter-frequency domain and, thus, a transformation to absolute (true) frequency domain is necessary. Indeed, the former characteristic should be emphasised, since the 
present work and that of [12] yield sea state estimates with CPU times in the order of only a few seconds.

The article is composed as follows: After the introduction, Sections 2 and 3 outline the theory in terms of the governing equations and the solution strategy, including the actual estimation algorithm. The algorithm has some restrictions, mentioned in Section 4 together with other practicalities. The estimation procedure's performance is comprehensively investigated through a number of test cases consisting of (artificial) simulations of measurement data, Section 5 , and the associated results and discussions are given in Section 6. The estimation algorithm is also tested using full-scale data, Section 7, obtained from sea trials with the research vessel R/V Gunnerus. Finally, Section 8 presents conclusions and suggests further work.

\section{Spectral analysis and fundamental equations}

The wave-induced (motion) responses of a ship in an irregular, short-crested seaway are considered. It is assumed that the responses are linear with the incident waves, and the speed and (mean) heading of the vessel relative to the waves are $U$ and $\chi \in$ $[0,360$ [ deg., respectively, with $\chi=180 \mathrm{deg}$. being head sea. The wave energy is distributed according to a directional wave energy spectrum $S\left(\omega_{0}, \mu\right)$ where $\omega_{0}$ is the absolute (wave) frequency and $\mu \in]-180,180] \mathrm{deg}$. is the angle describing the directional variation of the spectral energy relative to an axis parallel with the vessel's centreline. For a given vessel speed, the set of wave frequency and relative heading implies a certain (and unique!) encounter frequency $\omega_{e}$ determined by the Doppler Shift,

$$
\omega_{e}=\omega_{0}-\omega_{0}^{2} \psi, \psi=\frac{U}{g} \cos \chi
$$

where $g$ is the acceleration of gravity. The Doppler Shift expresses a mathematical/physical elementary, but it is important to realise that the practical complications related to the Doppler Shift is by no means straight-forward to handle for wave-induced responses of an advancing ship in a seaway, as also mentioned in various textbooks [e.g. 13, 14, 15].

The estimation problem is formulated in the frequency domain through spectral analysis assuming stationary conditions. The linear relationship between waves and waveinduced vessel responses (here only heave, roll and pitch are considered) is given by the 
complex-valued motion transfer functions $X_{i}(\ldots)$, which can be calculated using hydrodynamic software (e.g. strip theory and panel codes) and/or obtained by measurements. In a short-crested, stationary seaway it holds that,

$$
R_{i j}\left(\omega_{e}\right)=\int X_{i}\left(\omega_{e}, \mu+\chi\right) \overline{X_{j}\left(\omega_{e}, \mu+\chi\right)} S_{e}\left(\omega_{e}, \mu\right) d \mu
$$

where $R_{i j}\left(\omega_{e}\right)$ is the complex-valued cross spectrum for a pair $(i, j)$ taken among the heave $(z)$, roll $(\phi)$, and pitch $(\theta)$ responses $i, j=\{z, \phi, \theta\} ; \overline{X_{j}(\ldots)}$ is the complex conjugate of the transfer functions in heave, roll and pitch for wave heading $(\mu+\chi)$ relative to the single waves from direction $\mu . S_{e}\left(\omega_{e}, \mu\right)$ is the wave spectrum ordinate as observed from the advancing ship; note that index 'e' is used to emphasise that the ordinate refers to the encounter domain. As an assumption, $S_{e}\left(\omega_{e}, \mu\right)$ is represented by the product between a point spectrum $E\left(\omega_{e}\right)$ and a directional spreading function $\varphi(\mu)$,

$$
S_{e}\left(\omega_{e}, \mu\right)=E\left(\omega_{e}\right) \varphi(\mu)
$$

Consequently, Eq. (2) is rewritten,

$$
R_{i j}\left(\omega_{e}\right)=E\left(\omega_{e}\right) \int X_{i}\left(\omega_{e}, \mu+\chi\right) \overline{X_{j}\left(\omega_{e}, \mu+\chi\right)} \varphi(\mu) d \mu
$$

The spreading function, e.g. [13], is taken as

$$
\varphi(\mu)=A(s) \times \cos ^{2 s}\left(\frac{\mu}{2}\right), A(s)=K \cdot \frac{2^{2 s-1} \Gamma^{2}(s+1)}{\pi \Gamma(2 s+1)}
$$

where $\Gamma$ denotes the Gamma function, and $s$ is the spreading parameter. The spreading function is obliged to fulfill $\int_{\mu_{1}}^{\mu_{2}} \varphi(\mu) d \mu \equiv 1$ from which the value of $K$ is determined for a given pair of directions $\left(\mu_{1}, \mu_{2}\right)$ that confines the directional spreading. If $\left(\mu_{1}, \mu_{2}\right)=$ $(-180,180)$ deg., then $K=1$.

The cross spectra $R_{i j}\left(\omega_{e}\right)$ can be calculated for sets $(i, j)$ of response measurement time series by using a cross power spectral density function, e.g., cpsd in MATLAB ${ }^{\circledR}$. An example of corresponding sets of cross spectra $R_{i j}\left(\omega_{e}\right)$ calculated from three measured time history recordings is shown in Figure 1 taken from Brodtkorb et al. [12]. From the plots/spectra it is observed that $R_{i j}\left(\omega_{e}\right)$ is complex-valued for $i \neq j$, and that corresponding cross-spectra pairs, or off-diagonal pairs, are complex conjugate, i.e. $\operatorname{I} m\left(R_{i j}\right)=-\mathcal{I} m\left(R_{j i}\right)$, which is a property that can be used to infer about the incident 
wave direction as seen later. The individual off-diagonal complex-valued spectra in Figure 1 can be given as corresponding real-valued pairs of amplitude- and phase-spectra by simply calculating the modulus and phase, respectively, for each frequency component of the given off-diagonal spectrum. For instance, the amplitude spectrum of the coupled motion of heave and roll is,

$$
\left|R_{z \phi}\left(\omega_{e}\right)\right|=\sqrt{\left[\mathcal{R} e\left(R_{z \phi}\left(\omega_{e}\right)\right]^{2}+\left[\mathcal{I} m\left(R_{z \phi}\left(\omega_{e}\right)\right]^{2}\right.\right.}
$$

and it is noted that the 'amplitude spectrum' has a similar meaning as the three diagonal spectra of heave, roll, and pitch, respectively, in Figure 1; namely, the amplitude spectrum represents the distribution of 'power' with frequency of the particular (coupled) motion component. Consequently, a total of six independent power (and three phase) spectra can be computed from the three measured motion components heave, roll, and pitch.

Rather than solving Eq. (4) with complex-valued spectra, it is decided (for numerical stability reasons) to solve the equation by introducing instead the six power spectra,
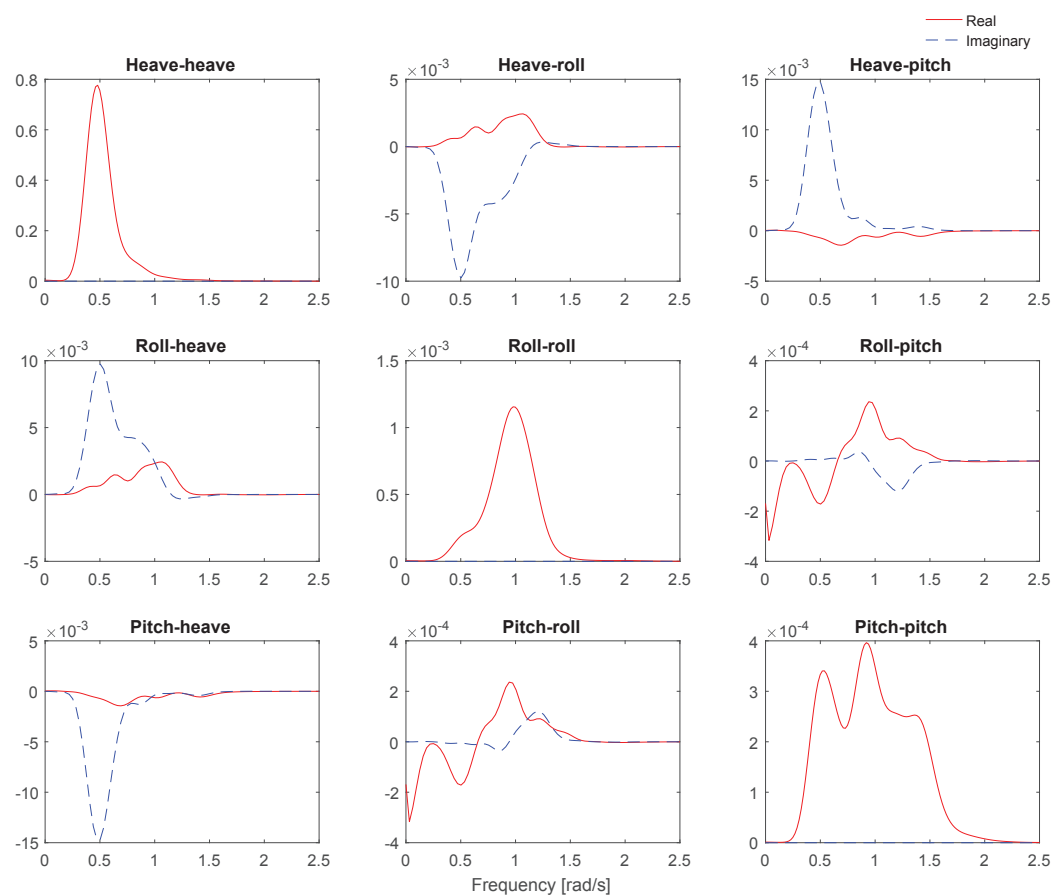

Figure 1: Cross spectra $R_{i j}$ calculated from measured responses in heave [m], roll [rad.] and pitch [rad.] of the research vessel R/V Gunnerus. Brodtkorb et al. [12]. 
and to leave phase information/equations out in the first step. Thus, the six governing equations of the estimation problem read,

$$
\left|R_{i j}\left(\omega_{e}\right)\right|=E\left(\omega_{e}\right) \int\left|X_{i}\left(\omega_{e}, \mu+\chi\right) \overline{X_{j}\left(\omega_{e}, \mu+\chi\right)}\right| \varphi(\mu) d \mu
$$

formed by the pairs of motion components $(i, j)$, which are $(z, z),(\phi, \phi),(\theta, \theta),(z, \phi)$, $(z, \theta)$, and $(\phi, \theta)$. In Eq. (7), the left-hand side is obtained through measurements while the right-hand side is obtained through theoretical calculations combining available knowledge about the motion transfer functions together with information about the wave energy spectrum.

It is important to note that Eq. (7) is formulated in the encounter-frequency domain $\left(\omega_{e}\right)$. However, the motion transfer functions of a vessel are determined for a set of absolute wave frequencies $\left(\omega_{0}\right)$, and it is therefore necessary to introduce the Doppler Shift, Eq. (1), when solving Eq. (7) for the unknown point-wave energy spectrum $E\left(\omega_{e}\right)$. When a ship advances in following seas*, the Doppler Shift imposes a 1-to-3 mapping between encounter and absolute frequencies, since one encounter frequency may be "clocked" at three different absolute frequencies in certain conditions [16]. Turning to Eq. (7), this means that for any (discrete) encounter frequency - with the 'following sea conditions' fulfilled - the corresponding three absolute frequencies need to be simultaneously considered on the right-hand side of Eq. (7), as the assumption is that the three frequencies, i.e. wave components, will contribute "equally" to form the encountered wave component. Symbolically, the corresponding pairs of encounter and absolute frequencies are written as $\left\{\omega_{e} \rightleftarrows \omega_{01}\right\}$ and $\left\{\omega_{e} \rightleftarrows \omega_{01}, \omega_{02}, \omega_{03}\right\}$ for head seas and following seas, respectively. The final version of the governing equation system is therefore given by,

$$
\begin{aligned}
\left|R_{i j}\left(\omega_{e}\right)\right|=E\left(\omega_{e}\right) \int \mid & \left|\Phi_{i j}\left(\omega_{01}, \mu+\chi\right)\right|^{2} \varphi(\mu) d \mu \\
& +E\left(\omega_{e}\right) \int\left[\left|\Phi_{i j}\left(\omega_{02}, \mu+\chi\right)\right|^{2}+\left|\Phi_{i j}\left(\omega_{03}, \mu+\chi\right)\right|^{2}\right] \varphi(\mu) d \mu
\end{aligned}
$$

where $\left|\Phi_{i j}\left(\omega_{0 k}, \mu+\chi\right)\right|^{2}=\left|X_{i}\left(\omega_{0 k}, \mu+\chi\right) \overline{X_{j}\left(\omega_{0 k}, \mu+\chi\right)}\right|$, and it is stressed that the first line of the equation is considered for all conditions, while the second line applies

\footnotetext{
*In this article, the term 'following seas' is at many places used to cover everything from following waves to beam waves (not included), while 'head seas' covers beam waves (included) to head waves.
} 
specifically to following seas. However, in following seas, the inclusion of the individual contributions is conditional, depending on the value of the encounter frequency relative to the wave heading and the speed of the vessel.

As pointed out, the corresponding set of frequencies, encounter vs. absolute, is given by the Doppler Shift, and solving Eq. (1) for the absolute frequency yields for head seas,

$$
\omega_{01}=\frac{1-\sqrt{1-4 \psi \omega_{e}}}{2 \psi}, \quad \text { all } \omega_{e}
$$

and for following seas,

$$
\begin{array}{ll}
\omega_{01}=\frac{1-\sqrt{1-4 \psi \omega_{e}}}{2 \psi}, & \omega_{e}<\frac{1}{4 \psi} \\
\omega_{02}=\frac{1+\sqrt{1-4 \psi \omega_{e}}}{2 \psi}, & \omega_{e}<\frac{1}{4 \psi} \\
\omega_{03}=\frac{1+\sqrt{1+4 \psi \omega_{e}}}{2 \psi}, & \text { all } \omega_{e}
\end{array}
$$

\section{Solution strategy and estimation algorithm}

The wave spectrum estimate is calculated in two steps: an initial step concerned with the direct, or brute-force, solution of Eq. (8), and a second - post-processing step concerned with a (final) wave direction estimate and a transformation to absolute domain of the wave spectrum estimate.

\subsection{Brute-force solution}

The brute-force solution does not assume a wave spectrum shape, or parameterise it in any way. Instead, the spectrum estimate is based on the direct solution of the linear equation, Eq. (8), which is solved using an iterative scheme as follows:

$$
\begin{aligned}
\tilde{R}_{i j}\left(\omega_{e}\right) & =R_{i j}\left(\omega_{e}\right)-\hat{R}_{i j}\left(\omega_{e}\right) \\
\left.\hat{E}_{i j}\left(\omega_{e}\right)\right|_{\chi=\chi_{k}} & =\left.\hat{E}_{i j}\left(\omega_{e}\right)\right|_{\chi=\chi_{k}}+h \tilde{R}_{i j}\left(\omega_{e}\right) \\
\hat{R}_{i j}\left(\omega_{e}\right) & =\left.\hat{E}_{i j}\left(\omega_{e}\right)\right|_{\chi=\chi_{k}} \int \sum_{m=1}^{3}\left|\Phi_{i j}\left(\omega_{0 m}, \mu+\chi_{k}\right)\right|^{2} \varphi(\mu) d \mu
\end{aligned}
$$

for a given heading $\chi_{k}=[0,180]$ deg. $(\chi=180 \mathrm{deg}$. is incident waves head on $)$. The iteration is performed for any pair $(i, j)$ of motion components; herein taken as $(z, z)$, 
$(\phi, \phi),(\theta, \theta),(z, \phi),(z, \theta)$, and $(\phi, \theta)$, and noting that Eq. (11c) is calculated conditionally with due account for head sea vs. following sea conditions, as addressed in relation with Eq. (8). Furthermore, it should be noted that Eq. (8) is solved for the entire range of (encounter) frequencies considered; in principle, in a frequency-by-frequency approach for the discrete computational settings, see below. Lastly, making a note of a more general character, the formulation of the problem in an iterative scheme, and based on a residual calculation, is a novel idea [12] compared to other existing spectral estimation procedures [e.g. 4, 5, 17, 8, 18, 19]. Indeed, the residual type of solution strategy is what makes the present procedure extremely efficient.

The practical implementation of the iterative scheme is illustrated in Algorithm 1, which should be read with some supplementary remarks about:

- Discretisation. Wave headings and the set of encounter frequencies are discretised into $N_{\chi}$ and $N_{\omega_{e}}$ parts. Since the wave heading is unknown initially, a loop is made over all directions

$$
\tilde{\chi}_{k}=[0,180] \text { deg., } \quad k=1: N_{\chi}
$$

- Initialisation. The estimate of the (encounter) wave spectrum, and the estimate of the response spectrum are initially set to zero, $\left.\hat{E}_{i j}\left(\omega_{e}\right)\right|_{\chi=\chi_{k}}=0$ and $\hat{R}_{i j}\left(\omega_{e}\right)=0$. Then, compute the difference between the measured response spectrum and the estimated response spectrum $\tilde{R}_{i j}\left(\omega_{e}\right)=R_{i j}\left(\omega_{e}\right)-\hat{R}_{i j}\left(\omega_{e}\right)$. Note that initialisation and computation apply to the discrete set of $t$ any encounter frequencies.

- Doppler Shift. The given frequency is the encounter frequency $\omega_{e}$, "produced" from the cross-spectral analysis, whereas the absolute frequency $\omega_{0}$ is a function of it. The function $f\left(\omega_{e} \mid \chi, U\right)$ is a result of the Doppler Shift; explicit expressions are seen in Eq. (9) and Eqs. (10a)-(10c).

- Updates/adjustments. Use $\tilde{R}_{i j}\left(\omega_{e}\right)$ to make adjustments to $\left.\hat{E}_{i j}\left(\omega_{e}\right)\right|_{\chi=\chi_{k}}$ with step size $h>0$, and calculate the response spectrum estimate $\hat{R}_{i j}\left(\omega_{e}\right)$ again. Do this until a threshold is reached $\left|\tilde{R}_{i j}\left(\omega_{e}\right)\right| \leq \epsilon$, for $\epsilon>0$.

The output from Algorithm 1 is six wave spectrum estimates per direction, yielding 


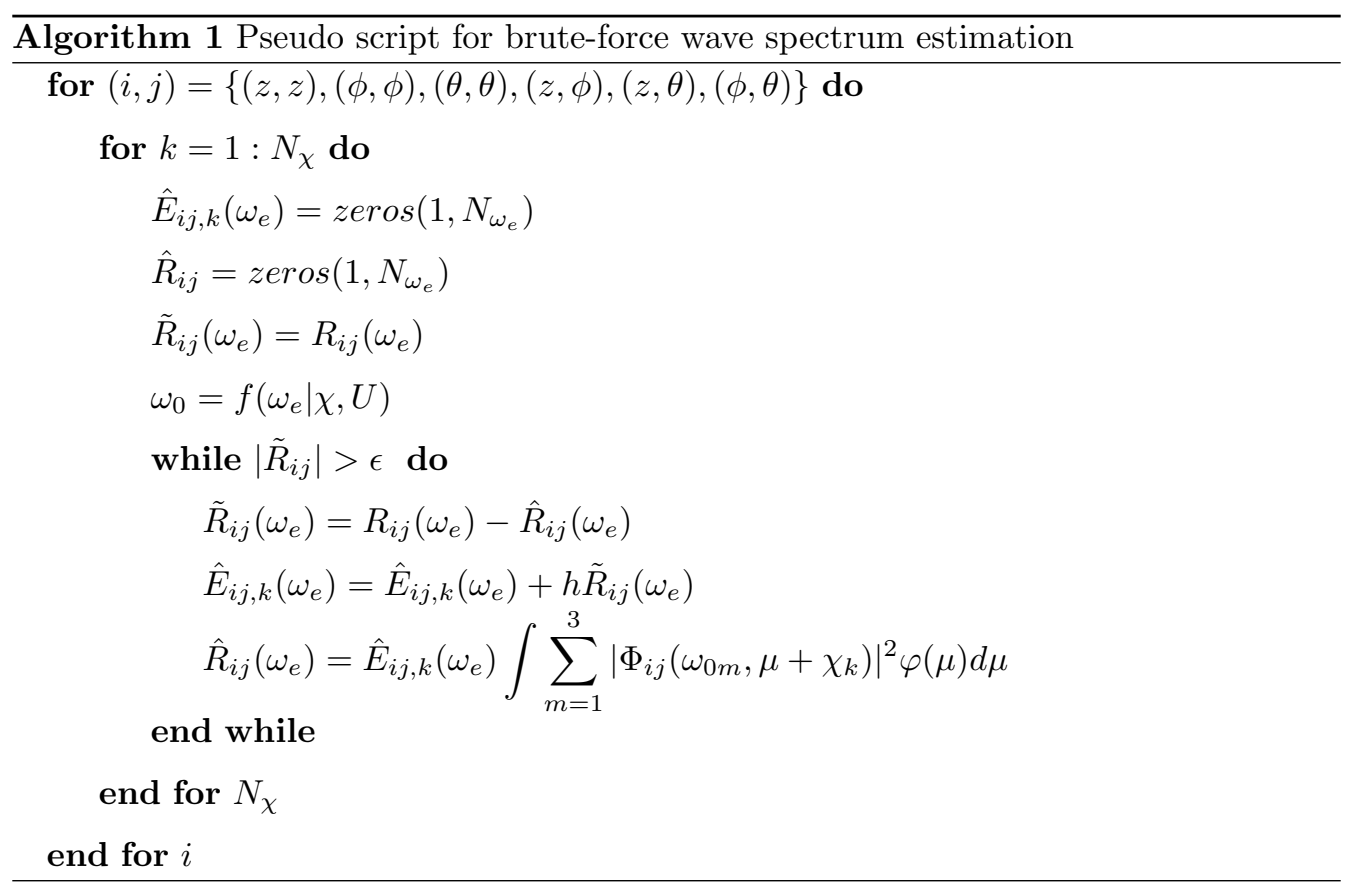

a spectrum estimate (block) matrix of dimension $6 \times\left(N_{\chi} \cdot N_{\omega_{e}}\right)$,

$$
\overline{\mathbf{E}}=\left[\begin{array}{cccc}
\hat{E}_{z z, 1}\left(\omega_{e}\right) & \hat{E}_{z z, 2}\left(\omega_{e}\right) & \ldots & \hat{E}_{z z, N_{\chi}}\left(\omega_{e}\right) \\
\hat{E}_{\phi \phi, 1}\left(\omega_{e}\right) & \hat{E}_{\phi \phi, 2}\left(\omega_{e}\right) & \ldots & \hat{E}_{\phi \phi, N_{\chi}}\left(\omega_{e}\right) \\
\hat{E}_{\theta \theta, 1}\left(\omega_{e}\right) & \hat{E}_{\theta \theta, 2}\left(\omega_{e}\right) & \ldots & \hat{E}_{\theta \theta, N_{\chi}}\left(\omega_{e}\right) \\
\hat{E}_{z \phi, 1}\left(\omega_{e}\right) & \hat{E}_{z \phi, 2}\left(\omega_{e}\right) & \ldots & \hat{E}_{z \phi, N_{\chi}}\left(\omega_{e}\right) \\
\hat{E}_{z \theta, 1}\left(\omega_{e}\right) & \hat{E}_{z \theta, 2}\left(\omega_{e}\right) & \ldots & \hat{E}_{z \theta, N_{\chi}}\left(\omega_{e}\right) \\
\hat{E}_{\phi \theta, 1}\left(\omega_{e}\right) & \hat{E}_{\phi \theta, 2}\left(\omega_{e}\right) & \ldots & \hat{E}_{\phi \theta, N_{\chi}}\left(\omega_{e}\right)
\end{array}\right]
$$

noting that each component in Eq. (13) is a row vector of length $N_{\omega_{e}}$, i.e. $\operatorname{size}\left(\hat{E}_{i j, k}\left(\omega_{e}\right)\right)=$ $1 \times N_{\omega_{e}}$.

\subsection{Post-processed solution}

The matrix in Eq. (13) represents the brute-force solution to the wave estimation problem considered in Eq. (8). However, it is clear that the solution, as is, cannot be directly used, since (1) no estimate of the wave heading, equivalently relative wave direction, is given because sub-solutions exist for all (specified) directions on a half circle 
$[0,180]$ deg., (2) the solution is ambiguous with several sub-solutions (herein six) depending on the considered response, and (3) the sub-solutions are encounter-frequency wave spectra. Altogether, it is therefore necessary to post-process the brute-force solution, and the approaches for doing this are explained below. Points (1) and (2) must be addressed simultaneously, and this is addressed in the following subsection, while point (3) is addressed in Subsection 3.2.2.

\subsubsection{Wave heading estimation (1) and making the solution unique (2)}

The single wave spectrum estimates in Eq. (13) apply to the encounter domain and, hence, the estimates provide no information about the (true) distribution of wave energy in the absolute domain. Nonetheless, the spectra (Eq. 13) can be used to obtain an estimate of the total energy content of the wave system, since energy preserves irrespectively of the domain. In general, the total energy of a wave system can be given in terms of the significant wave height $H_{s}$, calculated from the area under the wave energy spectrum, see e.g. Eq. (21) in Subsection 5.2. Thus, the following $\left(6 \times N_{\chi}\right)$ matrix is obtained

$$
\overline{\mathbf{H}}_{s}=\left[\begin{array}{cccc}
\hat{H}_{s, z z}(1) & \hat{H}_{s, z z}(2) & \ldots & \hat{H}_{s, z z}\left(N_{\chi}\right) \\
\hat{H}_{s, \phi \phi}(1) & \hat{H}_{s, \phi \phi}(2) & \ldots & \hat{H}_{s, \phi \phi}\left(N_{\chi}\right) \\
\hat{H}_{s, \theta \theta}(1) & \hat{H}_{s, \theta \theta}(2) & \ldots & \hat{H}_{s, \theta \theta}\left(N_{\chi}\right) \\
\hat{H}_{s, z \phi}(1) & \hat{H}_{s, z \phi}(2) & \ldots & \hat{H}_{s, z \phi}\left(N_{\chi}\right) \\
\hat{H}_{s, z \theta}(1) & \hat{H}_{s, z \theta}(2) & \ldots & \hat{H}_{s, z \theta}\left(N_{\chi}\right) \\
\hat{H}_{s, \phi \theta}(1) & \hat{H}_{s, \phi \theta}(2) & \ldots & \hat{H}_{s, \phi \theta}\left(N_{\chi}\right)
\end{array}\right]
$$

where the single matrix elements are calculated by use of the individual components of Eq. (13), i.e. $\hat{H}_{s, i j}(k)$ is obtained from $\hat{E}_{i j, k}\left(\omega_{e}\right)$.

In a purely theoretical situation there will be just one column, say no. $k_{K}$ in Eq. (14), where all the six elements attain the same non-zero value; that is, the average of the values in the column is equal to the values of the single elements. Consequently, the hypothesis is that column no. $k_{K}$ yields the optimum estimate of the significant wave height and, at the same time, the relative (mean) wave heading will be $\hat{\chi}=\tilde{\chi}\left(k_{K}\right)$, cf. Eq. (12). In practice, it is highly unlikely that the described truly theoretical situation happens and, rather, the column (from Eq. 14) with the smallest variation in between the significant wave heights can be found. Thus, the given column, i.e. the discrete 
value of the heading representing the column, can be used as an estimate of the wave heading.* It now remains to make the wave spectrum estimate unique, as it should be realised that, to this point, six wave spectrum estimates $\hat{E}_{i j, k_{K}}\left(\omega_{e}\right)$ apply to column $k_{K}$; i.e. one for each motion component, cf. Eq. (13). Therefore, the 'optimum' wave spectrum estimate is taken as the average of the six spectrum estimates; noting that the average is calculated frequency-wise,

$$
\begin{aligned}
\hat{E}_{f i n a l}\left(\omega_{e}\right)=\frac{1}{6}\left(\hat{E}_{z z, k_{K}}\left(\omega_{e}\right)+\hat{E}_{\phi \phi, k_{K}}\left(\omega_{e}\right)+\hat{E}_{\theta \theta, k_{K}}\left(\omega_{e}\right)\right. & \\
& \left.+\hat{E}_{z \phi, k_{K}}\left(\omega_{e}\right)+\hat{E}_{z \theta, k_{K}}\left(\omega_{e}\right)+\hat{E}_{\phi \theta, k_{K}}\left(\omega_{e}\right)\right)
\end{aligned}
$$

On a related note, the 'averaging-approach' has similarities to the study by Nielsen and Stredulinksy [20] that discusses the importance in selecting the best combination of motion measurements. This referred study uses also a mean-value-based solution, where all relevant combinations of motion measurements are considered and used for wave estimation. However, a general discussion about response selection for shipboard sea state estimation is beyond the scope of the present work and, thus, not addressed any further, but another useful study in this context has been given by Nielsen et al. [21].

It is a concern of the selection procedure described above that the obtained wave heading estimate will not necessarily be the (correct) optimum, since the selection procedure includes no distinction between incident waves on the port side and on the starboard side. The means to accommodate this problem is to make direct use of the complex-valued off-diagonal spectra, cf. Figure 1. Specifically, the imaginary parts of the off-diagonal elements should be considered, as these parts contain the necessary information because they are measures of the phases between the (coupled) motions. Hence, with reference to the fundamental equation, see Eq. (4), and the derived equation system Eq. (7) considering the six power spectra, three additional equations are considered:

$$
\mathcal{I} m\left[R_{i j}\left(\omega_{e}\right)\right]=E\left(\omega_{e}\right) \int \mathcal{I} m\left[X_{i}\left(\omega_{e}, \mu+\chi\right) \overline{X_{j}\left(\omega_{e}, \mu+\chi\right)}\right] \varphi(\mu) d \mu
$$

formed by the pairs of motion components $(i, j)$, which in this case are $(z, \phi),(z, \theta)$, and $(\phi, \theta)$, respectively. Obviously, Eq. (16) needs to be implemented in the same way

*In Appendix A, a small numerical example outlines how the (initial) wave heading estimate is selected. 
as Eq. (8), taking into account the practical complications in following sea. However, in contrast to the brute-force solution, cf. Algorithm 1, Eq. (16) is not solved for the point-wave spectrum $E\left(\omega_{e}\right)$ as the unknown, but for the wave heading instead. This is done by stepping through a discretised set of headings $\tilde{\chi}_{\kappa}, \kappa=1,2, \ldots, \varkappa$ on the full circle $[0,360[$ deg. and calculating, for each heading, the right-hand side of Eq. (16), using the optimum wave spectrum estimate given by Eq. (15). The calculated right-hand side of Eq. (16) can be subtracted from the left-hand side resulting in an error estimate $\varepsilon_{\kappa}^{2}$ for the particular heading $\tilde{\chi}_{\kappa}$. The error estimate is defined in the least squares sense using the $L_{2}$ norm, and the (final) optimum wave heading is thus found for the heading $\tilde{\chi}_{\kappa}$ where $\varepsilon_{\kappa}^{2}$ attains its minimum,

$$
\min _{\tilde{\chi}_{\kappa}} \varepsilon_{\kappa}^{2} \equiv \min \left\|\mathcal{I} m\left[R_{i j}\left(\omega_{e}\right)\right]-f\left(\tilde{\chi}_{\kappa}\right)\right\|^{2}
$$

noting that the right-hand side of Eq. (16) has been written symbolically as $f\left(\tilde{\chi}_{\kappa}\right)$.

Clearly, the estimate of the optimum wave heading is made in a rather brute-force approach. This choice is made to keep the overall estimation procedure consistent and to be of a 'practical engineering' character, although it would be easy to obtain the optimum heading through a strict optimisation formulated through a cost function.

\subsubsection{Transformation to absolute domain (3)}

The wave spectrum estimate, Eq. (15), applies to a (particular) set of encounter frequencies. Consequently, it is necessary to transform the spectrum estimate from encounter to absolute domain, for which purpose a specific transformation procedure, developed in an interrelated study by Nielsen [16], is introduced. Thus, as the very final step, the optimum wave spectrum estimate $\hat{E}_{f i n a l}\left(\omega_{e}\right)$ undergoes transformation,

$$
E\left(\omega_{0}\right)=g\left(\hat{E}_{\text {final }}\left(\omega_{e}\right) \mid \tilde{\chi}_{\kappa}, U\right)
$$

where $g(\ldots)$ is the mapping-function [16] which consistently transforms the estimated wave spectrum from encounter to absolute domain. Note that the given variables and parameters are the set of encounter frequencies $\omega_{e}$, vessel forward speed $U$, and estimated wave heading $\tilde{\chi}_{\kappa}$ obtained from Eq. (17). 


\section{Practicalities}

To this point the estimation procedure has been presented for its fundamental concepts and the associated equations. Thus, it remains as the main task to evaluate the estimation algorithm. The evaluation will firstly be performed using perfectly controlled settings in terms of computational simulations, and, later, with sea trials data. However, before the data and the estimating performance are studied, a few practical aspects of the estimation procedure are discussed.

\subsection{Limitations}

Due to a solution strategy relying on a residual calculation through an iterative scheme, the estimation procedure provides a point spectrum; initially obtained in the encounter domain and subsequently transformed to the absolute domain with due account for vessel speed and (mean) wave direction. Described by a point spectrum, the wave system is inherently considered as unidirectional, but short-crested waves are "imposed" into the solution by an overlaid directional spreading function. Nonetheless, the solution, or the wave spectrum estimate, is restricted from handling truly crossed-sea conditions where wind waves from one direction occurs at the same time as swells from another (very) different direction. In short, the estimation procedure is limited to deal with mixed seas (wind waves + swells) from the same direction. Or, said equivalently, the procedure facilitates estimation of crossed-seas, but the final estimate will be given as a point spectrum* with no distinction between the directions.

Like for all other shipboard estimation techniques, the current estimation procedure will be limited to estimate wave components at a certain frequency band. This limitation is due to the general characteristic of a ship being a low-pass filter. Hence, the algorithm will work best for wave lengths larger than some specific value relative to the ship length (and breadth); which obviously are case-specific parameters.

\subsection{Spectral calculations}

It has already been stressed that stationary conditions are considered/assumed exclusively in this study, which means that spectral analysis of the motion recordings, given

* Likely, the wave spectrum estimate will be fairly accurate if the incident directions of wind waves and swells are not too different. A hypothesis, however, that needs attention in any future work. 
as time series, will provide reliable results. In practice, it is difficult to define exactly when conditions are no longer (statistically) stationary, implying that any outcome from spectral analysis will be unreliable. Consequently, it should be interesting to consider, more carefully, in which conditions 'standard' spectral analysis cannot be applied for its particular purpose, i.e. to provide (cross) response spectra, in the context of shipboard sea state estimation. At the same time, it should be mentioned that elaborate means and procedures exist for conducting spectral analysis in nonstationary conditions and, potentially, it should therefore be possible to apply the studied (spectral) estimation procedure even when conditions are not stationary. However, these types of work are beyond the scope of the present study, and herein it suffices to note that several methods/tools are available to carry out the spectral analysis in case of stationary data.

By nature, ocean wave spectra are smooth in their frequency-wise distribution (and as well in their directional-wise distribution), and it is therefore necessary to work with smoothed versions of the set of response spectra. In the numerical studies analysed later, smoothing is imposed by a Parzen window applied with a $50 \%$ overlap on the full range of frequencies from the FFT. The resulting spectra are specified on 600 frequencies for a set of lower and higher cut-off frequencies $f_{\text {low }}=0.0008 \mathrm{~Hz}$ and $f_{\text {high }}=1.0 \mathrm{~Hz}$, respectively, and, consequently, the wave spectrum estimate(s) apply to the same range of encounter frequencies. Indeed, it is possible to work with such a fine frequency resolution only because of a highly computationally efficient estimation algorithm. In real-case practices, however, the resolution should be significantly lower in order to optimise computational speed; taking note that, at some point, the wave estimates will be affected if the resolution is too coarse.

\subsection{Wave spectrum transformation algorithm}

The details of the wave spectrum transformation algorithm outlined in [16] will not be dealt with herein. Anyhow, a few remarks about the algorithm are noteworthy. Briefly said, the algorithm is based on a scaling approach that assures preservation of energy at corresponding sets of encounter and (true) absolute frequencies. Thus, a set of scaling ratios apply to specific absolute frequencies, obtained through the Doppler Shift of given encounter frequencies, and multiplication between the scaling ratios and the encounter wave spectrum ordinate will make the transformed absolute wave spectrum available. It 
happens that spectral ordinates are erroneously transformed from the encounter domain to a high-frequency range of the absolute spectrum; despite 'consistent' multiplication with the spectral ratios. Consequently, the transformation algorithm introduces a tailfitting which makes sure that for frequencies higher than a user-defined value, the tail of the transformed spectrum follows that of a Bretschneider spectrum. In the particular case studies presented later, this values is taken as $0.25 \mathrm{~Hz}$.

\section{Case studies using simulated motions}

The performance of the estimation procedure (Sections 2 and 3) is evaluated using artificial time series data generated through computational simulations. In this setting, exact knowledge is available about the true wave energy spectrum and associated sea state parameters and, hence, it is easy to conduct comparative studies with the corresponding wave spectrum estimate obtained by the estimation procedure.

\subsection{Vessel data and motion transfer functions}

Time series simulation of the motion components \{heave, roll, pitch\} has been performed for an example ship with data given in Table 1. The example ship is identical to the research vessel $R / V$ Gunnerus, owned and operated by the Norwegian University of Science and Technology. R/V Gunnerus is also studied in Section 7, where full-scale motion recordings obtained through sea trials are studied.

In the simulation study, the same set of motion transfer functions is used to both generate the measurement time series and to subsequently estimate the wave spectrum with. Therefore, details about the transfer functions, including their absolute accuracy and agreement with the real hydrodynamics of the (full-scale) ship, are of minor importance. In the particular case, a set of closed-form expressions [22, 23] yields a good

\begin{tabular}{lr} 
Table 1: Main particulars of the example ship (R/V Gunnerus). \\
\hline Length, $L_{p p}$ & $28.9 \mathrm{~m}$ \\
Breadth, $B$ & $9.6 \mathrm{~m}$ \\
Draught, $T$ & $2.7 \mathrm{~m}$ \\
Block coefficient, $C_{B}$ & $0.56[-]$ \\
Waterplane coefficient, $C_{W P}$ & $0.84[-]$ \\
Displacement (mass), $\Delta$ & $417000 \mathrm{~kg}$ \\
Transverse metacentric height, $G M_{T}$ & $2.66 \mathrm{~m}$ \\
\hline
\end{tabular}


representation of the transfer functions considering heave, roll, and pitch. Moreover, the use of closed-form expression facilitates the possibility to make rapid calculations for updated operational conditions (e.g. Subsection 6.2). It is noteworthy, however, that the sea trials data, studied in Section 7, is indeed analysed using strip theory calculations based on the real hull geometry of the studied vessel.

\subsection{Wave scenarios (test cases)}

Various simulated test cases form the background for the performance evaluation of the estimation procedure. Each test case is represented by a given short-crested inputwave system characterised by a parameterised wave energy spectrum and its associated (true) integrated wave parameters such as significant wave height $H_{s}$, mean (wave) period $T_{m}$, and peak (wave) period $T_{p}$. Thus, the wave elevation and corresponding motion records can easily be generated for a ship advancing in the particular wave system, see Subsection 5.3 below.

An overview of the test cases (A, B, C) is seen in Table 2 which specifies the (absolute) input-wave parameters together with other operational parameters. It is noteworthy that two of the main test cases, A and B, differ only by the selected advance speed being $U=5.0$ knots and $U=10.0$ knots, respectively. The actual wave system, on the other hand, is exactly the same for the two cases, and so is the selected sets of mean headings $\chi_{0}$ relative to the wave system. The main purpose with the subcases of cases A and B is to evaluate the performance of the estimation procedure when the ship advances at different relative (mean) headings specified as $\chi_{0}=\{0,10, \ldots, 350\}$ deg. Notably, the concern is the procedure's ability to correctly estimate the wave system in following seas, and its ability to differentiate between incident waves on the starboard side or the port side. Note, at deep water conditions, the particular choice of mean period $T_{m}=6.5 \mathrm{~s}$

Table 2: Summary of test cases using a Bretschneider wave spectrum overlaid with a spreading function, where the latter has $s=4$ in every case.

\begin{tabular}{lccccc}
\hline Cases & $U[\mathrm{knots}]$ & $T_{m, 0}[\mathrm{~s}]$ & $T_{p, 0}[\mathrm{~s}]$ & $H_{s, 0}[\mathrm{~m}]$ & $\chi_{0}[\mathrm{deg}]$. \\
\hline A & 5.0 & 6.5 & 8.4 & 2.0 & $\{0: 10: 350\}$ \\
B & 10.0 & 6.5 & 8.4 & 2.0 & $\{0: 10: 350\}$ \\
C & 10.0 & $4.3+9.8(6.1)$ & $5.6+12.7$ & $2.0+2.0(2.8)$ & $\{0: 10: 350\}$ \\
\hline
\end{tabular}


corresponds to an absolute wave length $\lambda=\frac{g T_{m}^{2}}{2 \pi}=66.0 \mathrm{~m}$, i.e. $\lambda / L_{p p} \approx 2$. Thus, the wave system is of a wave-length regime where most of the wave energy is concentrated at wave lengths inducing reasonable response levels of the considered motion components \{heave, roll, pitch\}; an illustration of the given wave spectrum can be seen together with the set of motion transfer functions in Figure B.16 in Appendix B.

The reason to compose the test cases using two (different) vessel speeds is that fewer waves will "overtake" the vessel for the higher vessel speed ( $U=10$ knots) compared to the lower one, when the ship advances in following seas, and this fact may influence the outcome from the estimation procedure, as the physics behind the 1-to-3 relationship is indeed governed by the advance speed of the vessel (together with wave heading).

In addition to cases $\mathrm{A}$ and $\mathrm{B}$, one last test case, $\mathrm{C}$, representing a double-peaked wave system, is used to test the estimation procedure's performance in sea states with swells and wind sea occurring at the same time.

The listed wave scenarios in Table 2 are described by a Bretschneider (point) wave spectrum $S_{B}\left(\omega_{0}\right)$ overlaid with a spreading function $\varphi(\mu)$ (Eq. 5). That is, for the generation of the wave elevation and the corresponding motion records, the input-wave spectrum $S\left(\omega_{0}, \mu\right)$, also denoted the generating spectrum, is taken as:

$$
\begin{aligned}
S\left(\omega_{0}, \mu\right) & =S_{B}\left(\omega_{0}\right) \varphi(\mu) \\
S_{B}\left(\omega_{0}\right) & =173 \frac{H_{s}^{2}}{T^{4} \omega_{0}^{5}} \exp \left[-\frac{692}{T^{4} \omega_{0}^{4}}\right]
\end{aligned}
$$

where the characteristic period $T$ depends on which statistical period is given. The following substitutions apply: $T=T_{m}$ for the mean period $T_{m}, T=0.772 T_{p}$ for the peak period $T_{p}$, or by $T=1.086 T_{z}$ for the zero-upcrossing period $T_{z}$. Case $\mathrm{C}$ will be taken as the sum of two Bretschneider spectra with parameters as given in Table 2.

The generating spectrum depends on the input parameters (e.g., $H_{s}, T_{m}$ ) and for quantitative comparisons it is relevant to obtain the corresponding estimates. Thus, integrated wave parameters can be derived from the $\mathrm{n}$-th order spectral moments of a wave spectrum,

$$
\begin{aligned}
& m_{n}=\int_{0}^{\infty} \omega_{0}^{n} E\left(\omega_{0}\right) d \omega_{0} \\
& H_{s}=4 \sqrt{m_{0}}, \quad T_{m}=2 \pi \sqrt{\frac{m_{0}}{m_{1}}}, \quad T_{p}=\frac{2 \pi}{\omega_{p}}
\end{aligned}
$$


where $E\left(\omega_{0}\right)$ is given by Eq. (18), and $\omega_{p}$ is the frequency corresponding to the spectrum peak.

\subsection{Time history simulations}

The motion responses are simulated using standard procedure assuming Gaussian processes, e.g. [24], and a short description given in Appendix C. Based on the parameters of a particular test case, see Table 2, 20 sets of wave and motion measurements are generated. The need for several corresponding records, here 20, of wave and motion components is due to the fact that a statistical evaluation of the estimation procedure's performance is necessary, since a single, finite time history recording is just one out of the infinitely many that comprise the "complete" ensemble. The single time history records are 20 minutes long and made from $N=800$ wave components spaced non-equidistantly on the frequency interval $] 0,2 \pi]$ at $M=19$ wave directions using the spreading function (Eq. 5) with $s=4$. The time history simulations are generated at $10 \mathrm{~Hz}$, and, after adding white noise $(\mathrm{SNR}=20)$, the records are down-sampled to $2 \mathrm{~Hz}$ to artificially add measurement noise.

\section{Spectrum estimates based on simulated motions}

In this section, the performance of the estimation procedure is analysed and discussed. The case studies, see Table 2, have been presented in the preceding section, and the result will simply be the outcome of the estimation procedure when it is applied to the test cases. However, two overall subsets of results are considered, with the main subset reported in the following subsection that studies a situation where perfect knowledge about the hydrodynamic behaviour of the vessel in waves exists. That is, a situation where the motion transfer functions "by default" yield a perfect description of wavevessel interactions. As another situation, incomplete knowledge about the wave-vessel interactions is introduced to resemble a more realistic scenario. This situation can easily be studied by working with two different sets of motion transfer functions; one set for the time series generation and one set for the wave estimation process. 


\subsection{Perfect transfer functions}

In this part of the evaluation of the estimation procedure, the same set of transfer functions is used for the motion simulation and for the sea state estimation, respectively.

\subsubsection{Cases $A$ and $B$}

The specific outcome of the estimation procedure is a $(2 \mathrm{D})$ wave spectrum $E\left(\omega_{0}\right)$, and two (arbitrary) selections of estimated spectra taken from cases A and B are shown in Figures 2 and 3, respectively.

In the figures, each plot relates to a specific true wave heading, shown in the title of the plot, and the estimated wave heading is printed in the plot's legend. It is noteworthy that any plot is the result of just one out of the 20 sets of time history simulations representing the individual subcases/headings reported in Table 2. As such, it should be remembered that the single spectrum estimate may actually be estimating nicely the realised wave elevation process, i.e. its associated energy spectrum, for the specific (stochastic) realisation, although the spectrum estimate and the true generating (deterministic) spectrum are not fully alike for the specific realisation. Onwards, all comparisons of spectrum estimates, including wave parameter estimates, will be made
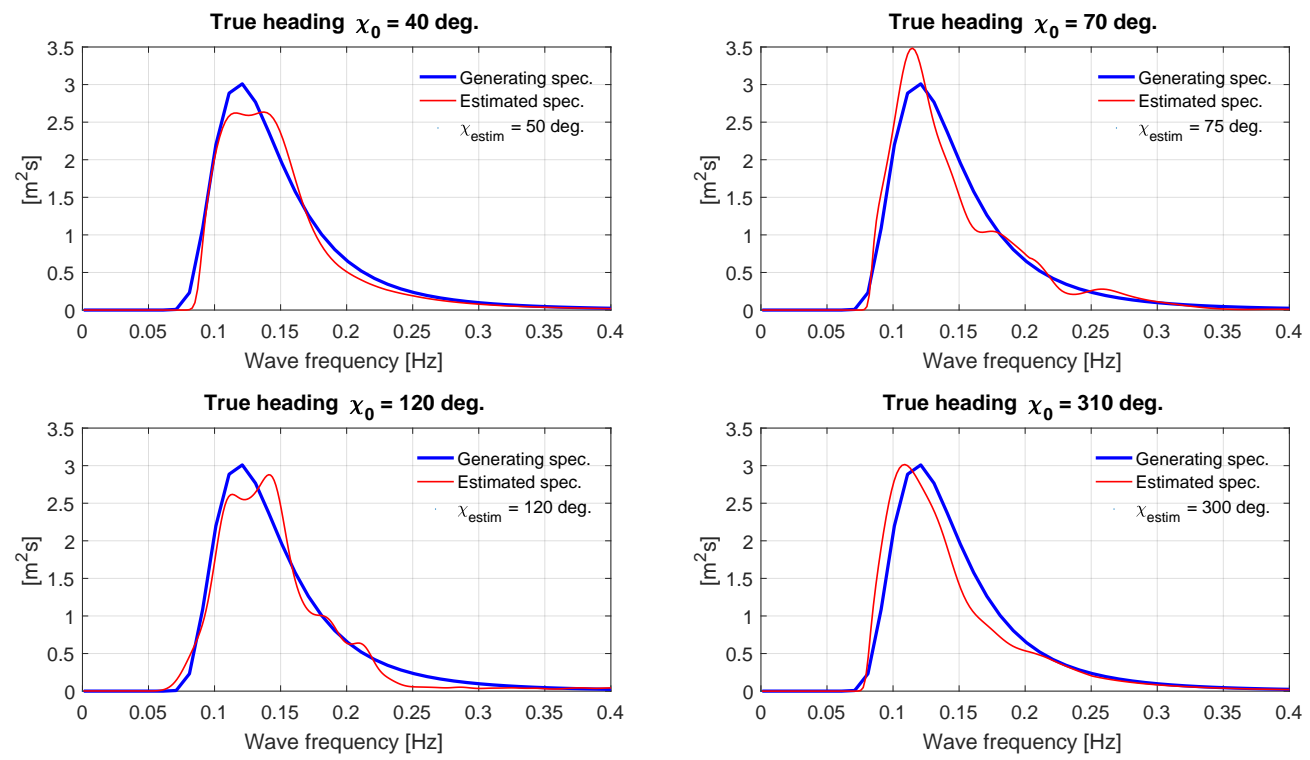

Figure 2: Examples of wave spectrum estimates for various wave headings in case A. The true and the estimated wave headings $(\chi)$ are included in the plot titles and legends, respectively. 
with respect to the (deterministic) values used to generate the particular wave scenario, despite the inconsistency in this - not always fair - comparison basis.

From the plots in Figures 2 and 3, it is evident that there is a good agreement between the true generating spectrum and the estimated one, including wave heading, in all of the considered comparisons. Although this observation is not entirely representative to every single set of time history recordings for every single subcase of cases A and B, Table 2, the general picture observed from Figures 2 and 3 resembles the overall trend of the spectrum estimates very well.

The trend, or the statistics, of the entire set of outcomes for cases A and B can be seen in Figures 4-7. Basically, the four figures contain the same sort of statistical information but relevant for the significant wave height (Fig. 4), the mean period (Fig. 5), the peak period (Fig. 6), and the (mean) relative wave heading (Fig. 7), with results shown for both cases $\mathrm{A}$ and $\mathrm{B}$ as the left-hand side plots $(U=5 \mathrm{knots})$ and the right-hand side plots $(U=10$ knots), respectively. The pairs of upper and lower plots in the figures present the same type of comparisons: The upper plot shows the average value of the outcome of estimates of the particular wave parameter considering all headings, and with
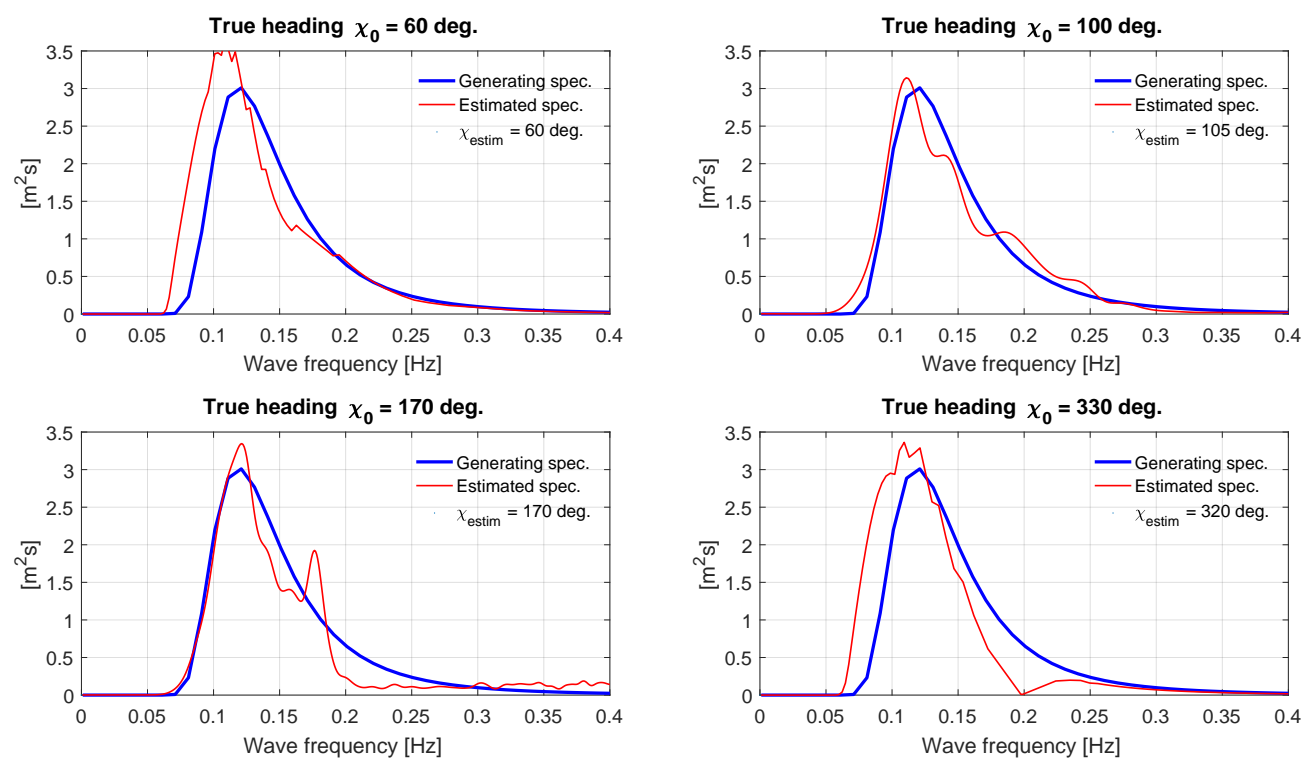

Figure 3: Examples of wave spectrum estimates for various wave headings in case B. The true and the estimated wave headings $(\chi)$ are included in the plot titles and legends, respectively. 
the average value based on the 20 sets of simulations for each heading. The error bar on the top of each column indicates plus/minus the standard deviation. The lower plot shows the statistics of the absolute deviation between estimates and the corresponding 'generating value' of the particular wave parameter. Irrespectively of the plot/figure it is decided to keep all comparative measures in absolute scale, since relative deviation/scale of wave parameters has, strictly speaking, only a meaning for the significant wave height; which is identical for cases A and B in this study.

Generally, the agreement (Figs. 4-7) between the estimates and the values used for the generation of the wave scenarios are good for all of the considered wave parameters, including the relative wave heading. Thus, it is observed that the average values (and the medians) are close to the 'generating values', with small variations around them although outliers occur here and there; and taking note that the peak period on average is estimated well but being the parameter with the most scatter in the results. The general
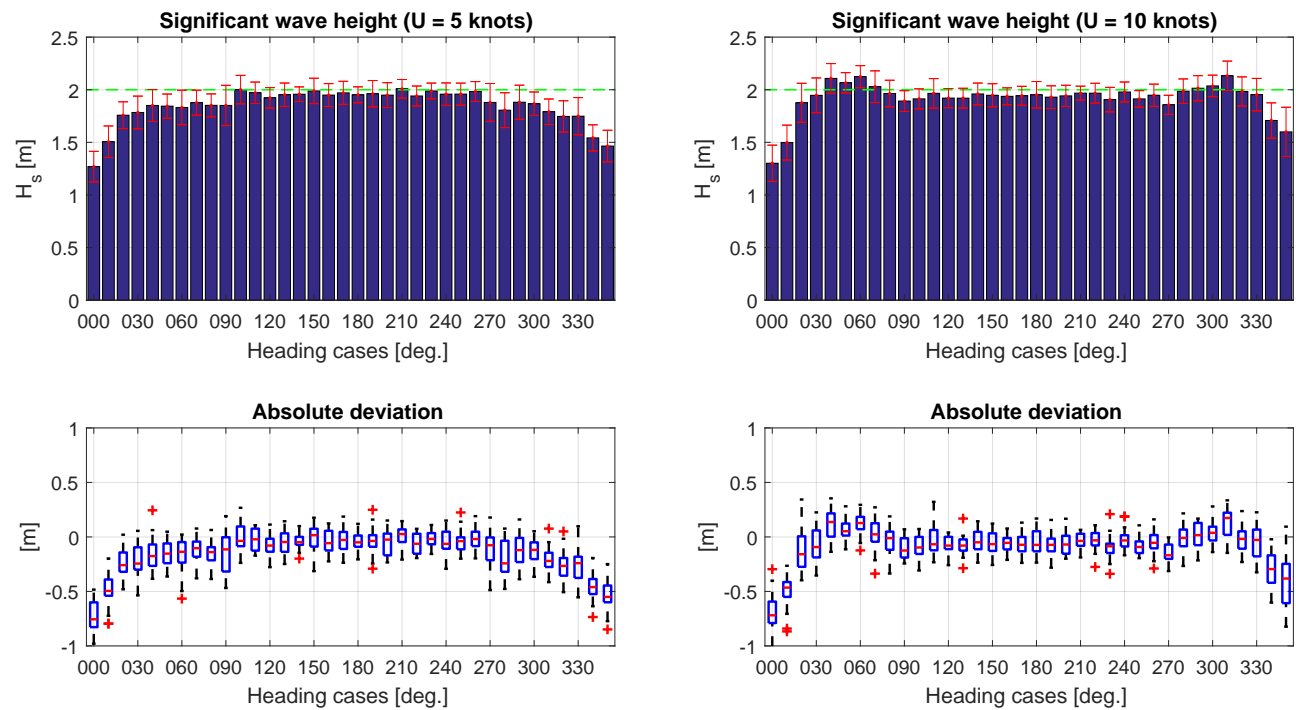

Figure 4: Upper plots: Estimates of significant wave height for all subcases (headings) of cases A (left-hand side) and B (right-hand side) with the value used to generate the theoretical spectrum indicated by the green dashed line. Lower plots: Deviations between estimates and 'generating value' shown as box plots. On each box, the central mark is the median, and the upper and lower edges of the box are the 25 th and 75 th percentiles, respectively. The whiskers extend to the most extreme data points which the algorithm considers not to be outliers, and the outliers are plotted individually. 
agreement drops a bit, however, when the ship is exposed to following sea conditions at incident wave angles closely in line with the vessel's centreline $(\chi \approx 0-10 \mathrm{deg}$. and $\chi \approx 350-360$ deg.).

Taking a more detailed look at the statistics, the results for the significant wave height (Fig. 4) reveal that the energy level almost consistently is slightly below the true level; an observation not limited to only following sea conditions. This sort of underestimation is a consequence of the filtering characteristic of a vessel in waves, making the ship less responsive to high-frequency waves, for what reason the observation/underestimation is expected. Indeed, this is one of the inherent and fundamental drawbacks of the wave buoy analogy and, as such, the observation applies to any other estimation technique reported in the literature; of course also for cases without forward speed [25]. Generally, the underestimation reduces as the wave period increases, which is a benefit to the wave buoy analogy considering the more severe wave scenarios.

As reported above, the most significant inaccuracies of the estimates occur in following to stern quartering sea conditions. Two specific outcomes of wave spectrum estimates are
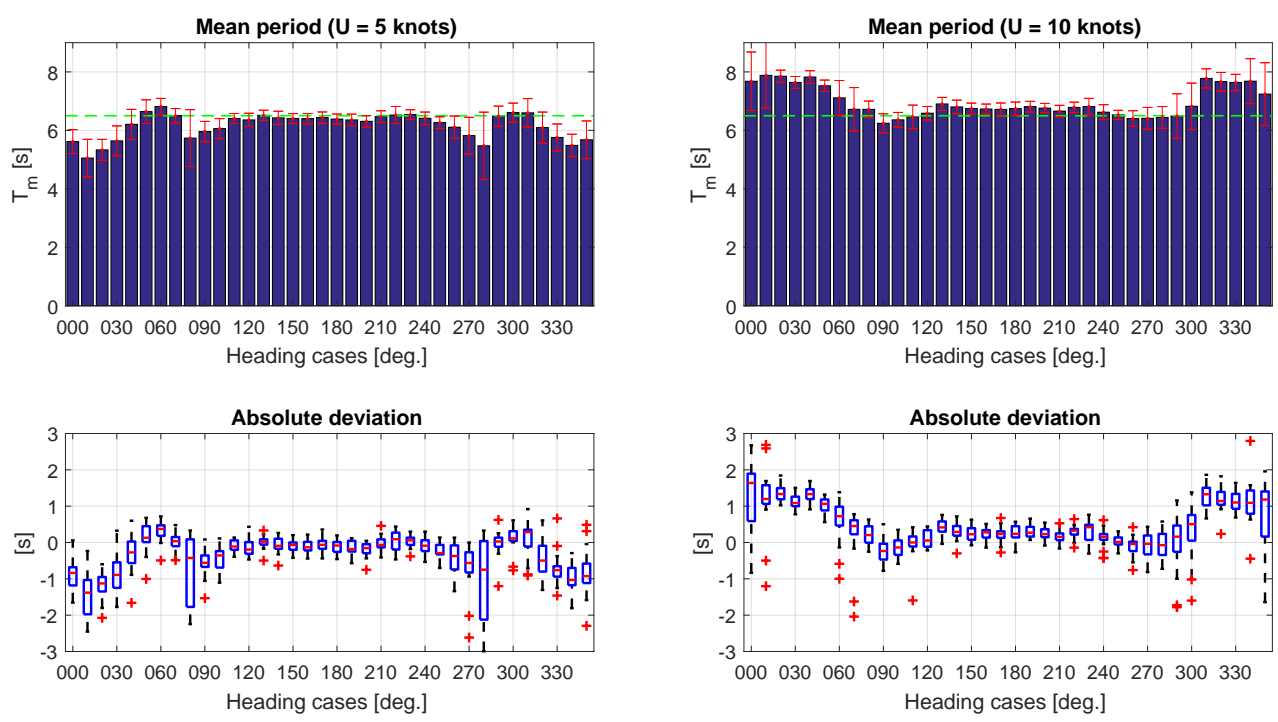

Figure 5: Upper plots: Estimates of mean period for all subcases (headings) of cases A (left-hand side) and B (right-hand side) with the value used to generate the theoretical spectrum indicated by the green dashed line. Lower plots: Deviations between estimates and 'generating value' shown as box plots; info is given in the caption of Fig. 4 . 
shown in Figure 8 for $U=10$ knots (case B) at $\chi_{0}=10$ deg. as true wave heading; noting that the specific spectrum estimates are quite representative for the remaining estimates at the heading (including $\chi=350 \mathrm{deg}$.); and applies also to the other vessel speed $U=$ 5 knots (case A). Efforts have tried to find the reason for the reduced agreement at the particular headings but no clear answer has been found. One plausible explanation could be related to the 1-to-3 relationship, i.e. the Doppler Shift, in following sea conditions, but - interestingly - very similar findings (not shown herein) apply for a situation without advance speed. Moreover, the 1-to-3 relationship is introduced also at the other headings in following sea conditions, notably $\chi=20-50 \mathrm{deg}$., where the estimates are as expected. It is therefore more likely that inaccuracies exist because of the actual hydrodynamic behaviour of the specific vessel, governed by hull geometry, when it is exposed to stern quartering waves (with or without advance speed), but investigations in this regard are left for future work.
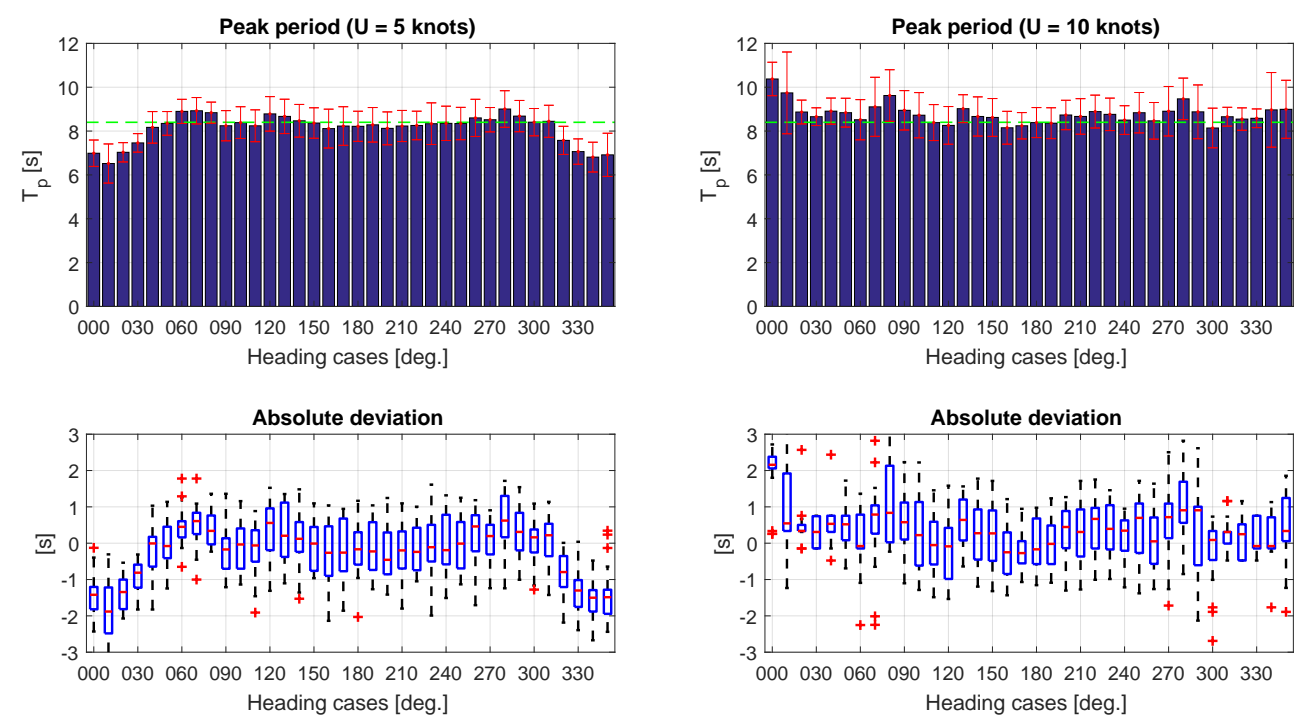

Figure 6: Upper plots: Estimates of peak period for all subcases (headings) of cases A (left-hand side) and B (right-hand side) with the value used to generate the theoretical spectrum indicated by the green dashed line. Lower plots: Deviations between estimates and 'generating value' shown as box plots; info is given in the caption of Fig. 4. 

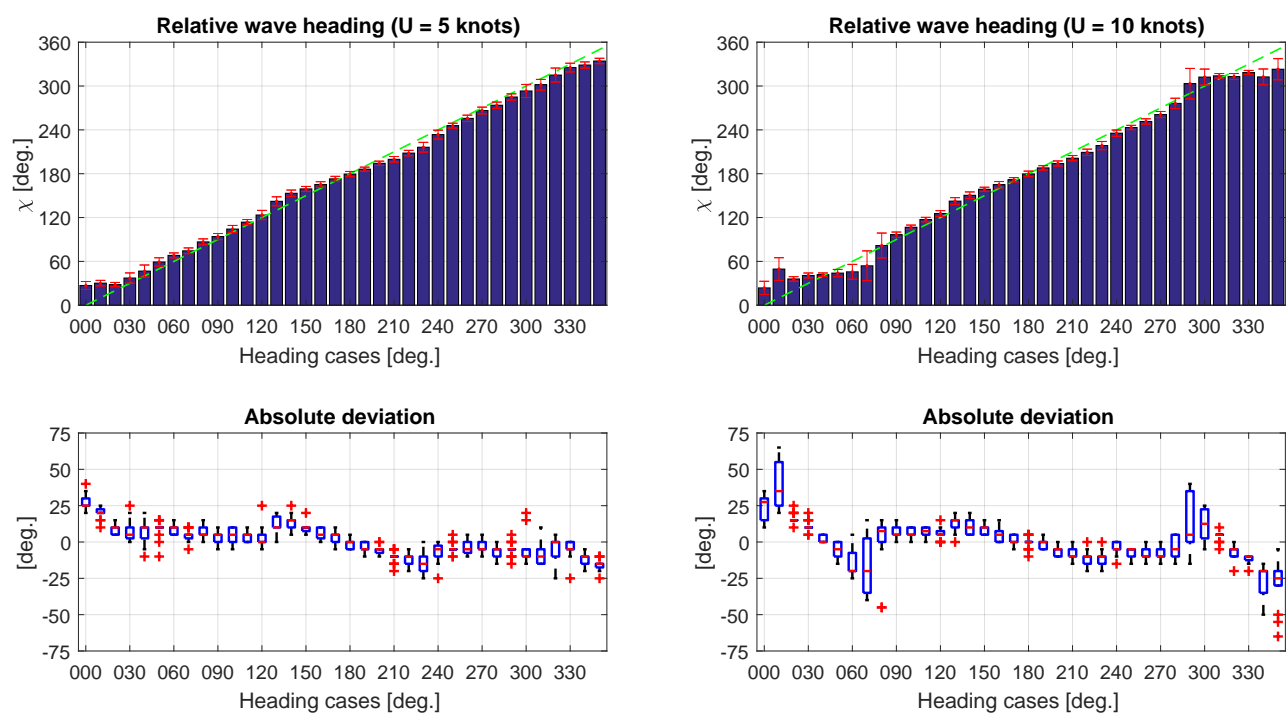

Figure 7: Upper plots: Estimates of (mean) relative wave heading for all subcases (headings) of cases A (left-hand side) and B (right-hand side) with the 'generating value' indicated by the green dashed line. Lower plots: Deviations between estimates and 'generating value' shown as box plots; info is given in the caption of Fig. 4.
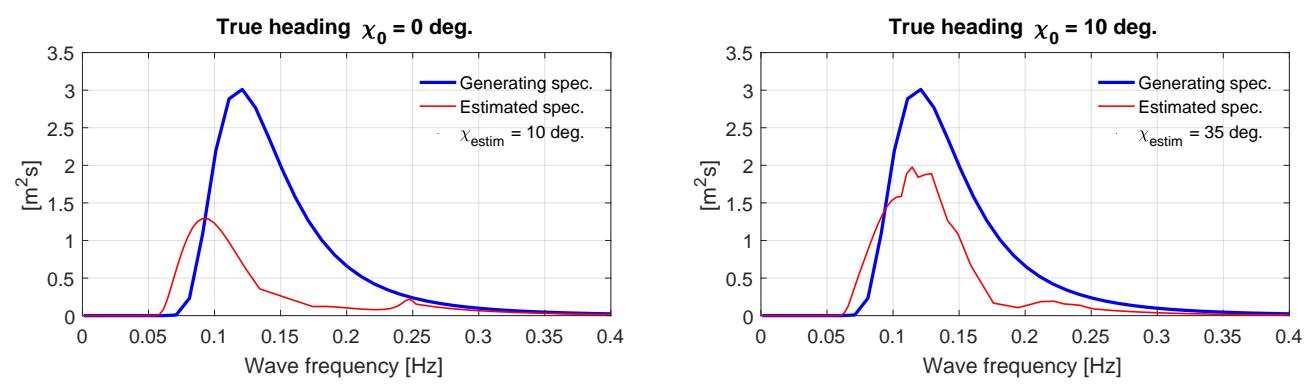

Figure 8: Examples of wave spectrum estimates in following sea for case B. The true and the estimated wave headings $(\chi)$ are included in the plot titles and legends, respectively.

\subsubsection{Case $C$}

In the last test case, $\mathrm{C}(U=10$ knots $)$, swells and wind seas occur at the same time, making the wave spectrum double-peaked. The entire set of statistical plots is included in Figure 9 showing average and median values, including variation, of significant wave height estimates (upper left plot-pair), peak period estimates of swell (upper right plotpair), peak period estimates of wind sea (lower left plot-pair), and wave heading estimates (lower right plot-pair), respectively. The statistics reveal somewhat similar findings, as 

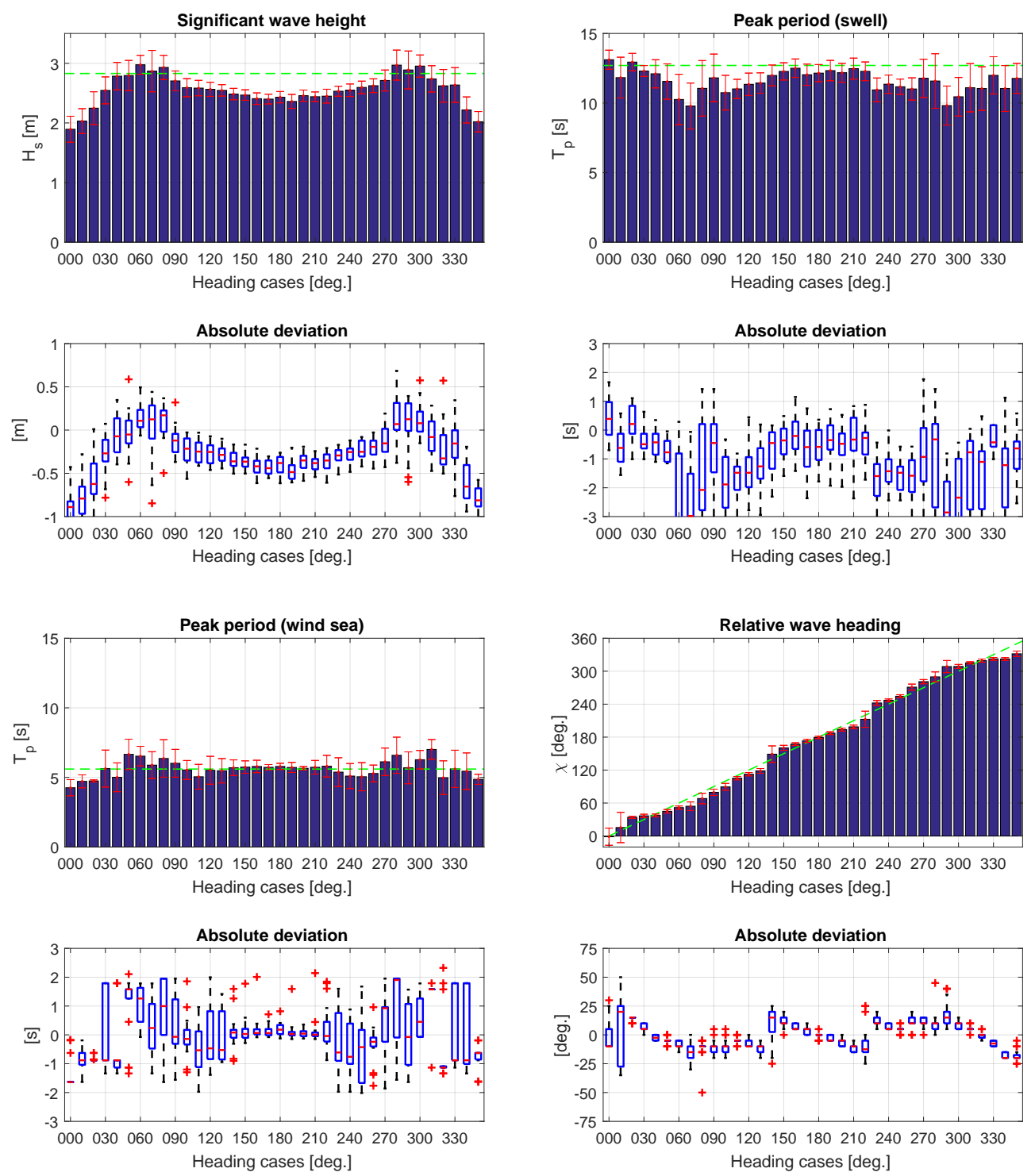

Figure 9: Estimates and deviations of significant wave height, peak periods for wind sea and swell, and relative wave heading, respectively, for all subcases (headings) of cases $\mathrm{C}$ with the value used to generate the theoretical spectrum indicated by the green dashed line. Deviations between estimates and true value shown as box plots. On each box, the central mark is the median, and the upper and lower edges of the box are the 25 th and 75 th percentiles, respectively. The whiskers extend to the most extreme data points which the algorithm considers not to be outliers, and the outliers are plotted individually. 
were observed in the previous cases (A and B), but generally with a lower agreement between the estimates and the corresponding values used for the generation of the wave scenarios, and substantially larger variation around the average values (and medians). An exception is however the wave heading estimates which are just as good in this case. It is noteworthy that at true headings $\chi_{0}=0$ deg. and $\chi_{0}=10$ deg., a few estimates are introduced with negative sign. That is, if the particular estimates were, say, $350 \mathrm{deg}$. the statistics are based on -10 deg. Some additional points to note from the plots are the following: The significant wave height is for all cases, except at wave headings just behind beam, lower than the 'generating values', which is a result of the filtering characteristic of the ship in waves. The "inconsistent" result (larger significant wave height) at headings just behind beam is not easily explained since it is not (necessarily) a result of fully correct spectrum estimates, which will be presented further below. The statistics concerning the peak periods of the swell and wind sea parts show an acceptable agreement, although the results are associated with rather large scatter. Moreover, it appears that at headings behind beam the peak periods are estimated closer together; i.e. the peak period of swell is too low and that of wind sea is too high. This observation is realised because the actual spectrum estimates are more blurred with a difficulty to distinctly detect (correctly) the two peaks of the wave system.

The findings mentioned above can be studied/confirmed by inspecting some of the actual spectrum estimates. Thus, for (true) wave headings $\chi_{0}=60 \mathrm{deg}$. and $\chi_{0}=180 \mathrm{deg}$. all 20 sets of realisations are included in Figure 10. Generally, the plots show a rea-
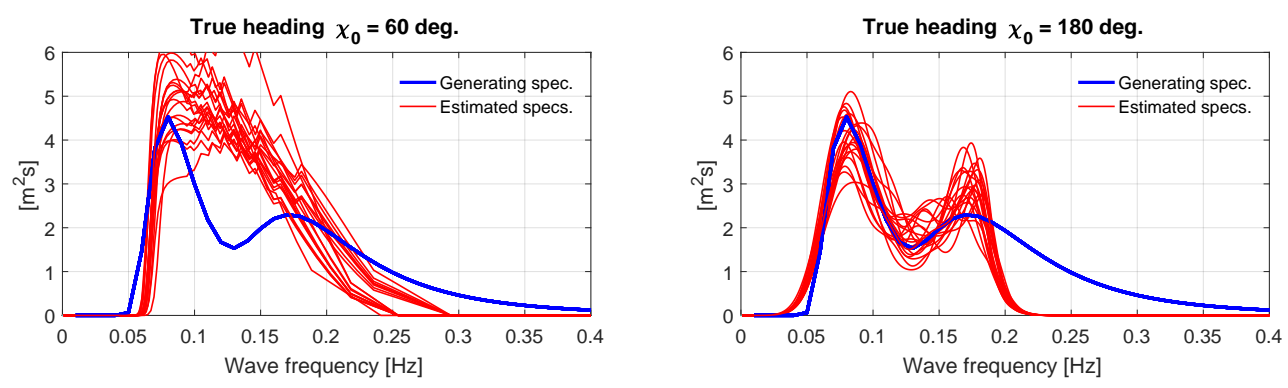

Figure 10: The entire set of wave spectrum estimates (total of 20) for headings $\chi_{0}=60$ deg. and $\chi_{0}=180 \mathrm{deg}$. as the left- and right-hand side plots, respectively. The estimated wave headings were, in most cases, close to the true values (see Fig. 9). 
sonable agreement between the spectrum estimates and the corresponding (generating) spectrum, capturing the most important part of the wave energy distribution. However, it is clear that the spectrum estimates are not as good as the findings were for cases A and B; notably problems occur for some of the 'behind-beam' sea conditions (around $\chi_{0}=60-90$ deg.) to detect the two individual peaks of the generating spectrum. It is also evident that the high-frequency part of the (true) wave energy distribution is not estimated correctly, which is a result of the filtering characteristic of the ship in waves.

\subsection{Imperfect transfer functions}

In this subsection, an imperfect set of motion transfer functions for the wave estimation process is imposed. More precisely, the transfer functions are calculated for a changed loading condition of the vessel, as "incomplete" knowledge is introduced simply by doing the calculations using as draught, $T_{\text {new }}=1.1 \cdot T_{0}$, as displacement, $\Delta_{\text {new }}=1.1 \cdot \Delta_{0}$, and as transverse metacentric height, $G M_{T, \text { new }}=0.9 \cdot G M_{T, 0}$, where the 0 -index relates to the original parameter values seen in Table 1. It must be emphasised, however, that the new set of transfer functions is used only in the wave estimation part, while the time history simulations are made using the original set of motion transfer functions, based on the input in Table 1. Otherwise, the operational conditions, including wave system and vessel speed, are exactly as case B, studied in the previous subsection, see also Table 2 .

The statistical outcomes of the entire set of spectrum estimates are presented in Figure 11. Indeed, the plots show that the estimates are still good, and by comparison to the right-hand side plots in Figures 4-7 the differences are barely visible. Basically, there are two (inter-related) ways to interpret this finding: 1) The estimation procedure is robust to changes in the applied motion transfer functions; 2) The particular example ship does not behave (very) differently when its loading condition is changed (slightly); or, strictly speaking, the calculated transfer functions $[22,23]$ exhibit little sensitivity to a change in the input parameters.

The findings for the imperfect set of motion transfer functions should actually be viewed in a wider perspective than merely as indications of robustness; either it be of the estimation procedure itself, or whether it means that the particular vessel and its associated hydrodynamic behaviour, represented by its motion transfer functions, 
exhibits little sensitivity to variations in the hull geometry and loading condition. Rather the key point is that, in real-case applications, it is useful to provide sea state estimates where an uncertainty measure, i.e. a "likeliness", is associated to the actual spectrum
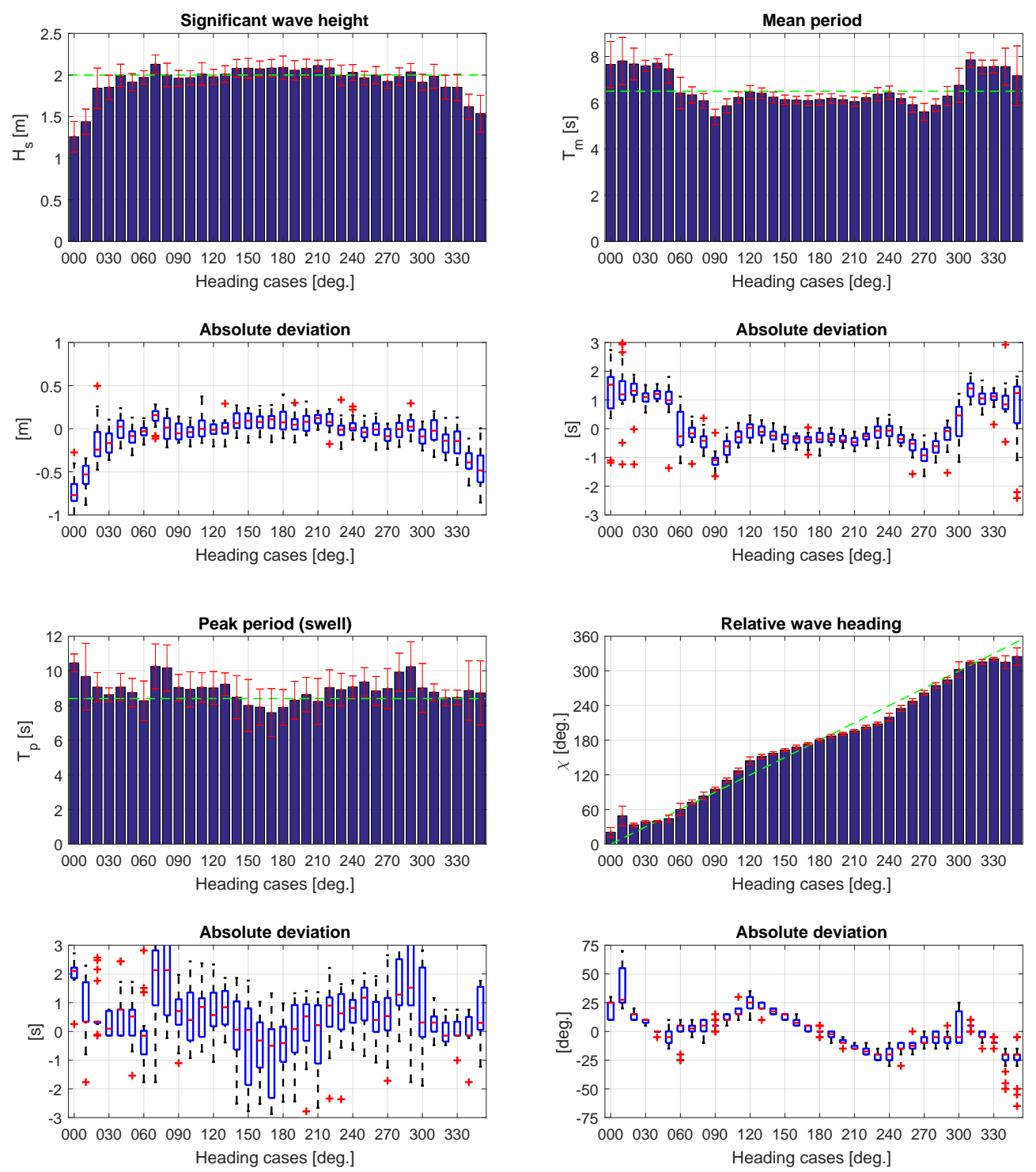

Figure 11: Estimates and deviations of significant wave height, mean periods, peak period, and relative wave heading, respectively, for all subcases (headings) of cases B using an imperfect set of motion transfer functions. 
estimate, or to the corresponding integrated wave parameters. Means to accomplish this has (conceptually) been discussed in the literature [e.g. 21, 26, 27] and a deeper discussion of the means is beyond the scope of the present article. Instead, it suffices to say that probabilistic calculations in this respect will require several sea state estimates - for the very same condition; governed by the exact same set of measurements, but using different sets of motion transfer function, where the input parameters are changed. Obviously, this means that the computational efficiency of the sea state estimation algorithm(s) must be very high. Indeed, this is so for the presented estimation procedure, since it is possible to obtain estimates in about 2-3 seconds (Intel(R) Core(TM) i7-4600U CPU @ $2.10 \mathrm{GHz}$ ), including also the cross spectral calculations of the time history recordings.

\section{Analysis of sea trials data}

Full-scale motion measurements have been collected during sea trials in 2013 with $\mathrm{R} / \mathrm{V}$ Gunnerus, see Table 1 for main dimensions and a photo of the vessel is shown in Figure 12. Originally, the sea trials were made to document the effect of a thruster retrofit [28], and, as part of this, DP tests as well as seakeeping runs were made. This section presents the outcome of the estimation algorithm when it is applied to the data of the seakeeping runs. The section leaves out detailed investigations and discussions of the results since it focuses entirely on the final outcome in terms of the (frequency-wise) wave

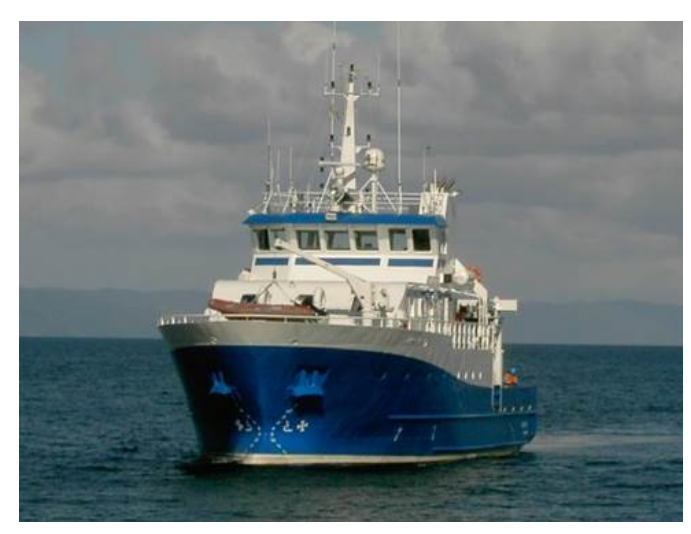

Figure 12: R/V Gunnerus 
spectrum and associated characteristic wave parameters. The input to the estimation algorithm is the three motion components heave, roll, and pitch, and the corresponding complex-valued transfer functions have been calculated with a 2D strip theory code, ShipX [29], using the exact hull geometry. As a related note, Brodtkorb et al. [12] present a comparison between the results of ShipX and the previously introduced closedform expressions (Subsection 5.1), for zero-advance speed, and a reasonable agreement exists.

The geographic run pattern of the tests is illustrated in Figure 13, where it is noted that the analysed motion recordings have been obtained on the four straight-line paths. On each path, indicated by IDs 16-19, course and engine power were held constant to secure stationary conditions, and the durations of the single straight-line runs were 2530 minutes. During the seakeeping tests the sea state was measured continuously by a free-floating wave buoy [30] deployed in the test area; but without information about the exact distance between the buoy and the individual run path. Table 3 presents the logged vessel speed together with vessel course relative to North (000 deg.), and the table also contains characteristic wave parameters obtained by the free-floating wave buoy, including significant wave height $\left(H_{s}\right)$, zero-upcrossing period $\left(T_{z}\right)$, peak period $\left(T_{p}\right)$, and mean wave direction $\left(\vartheta_{\text {mean }}\right)$. The mean wave direction is derived with consideration to the full frequency-directional distribution of energy, and the direction has been included

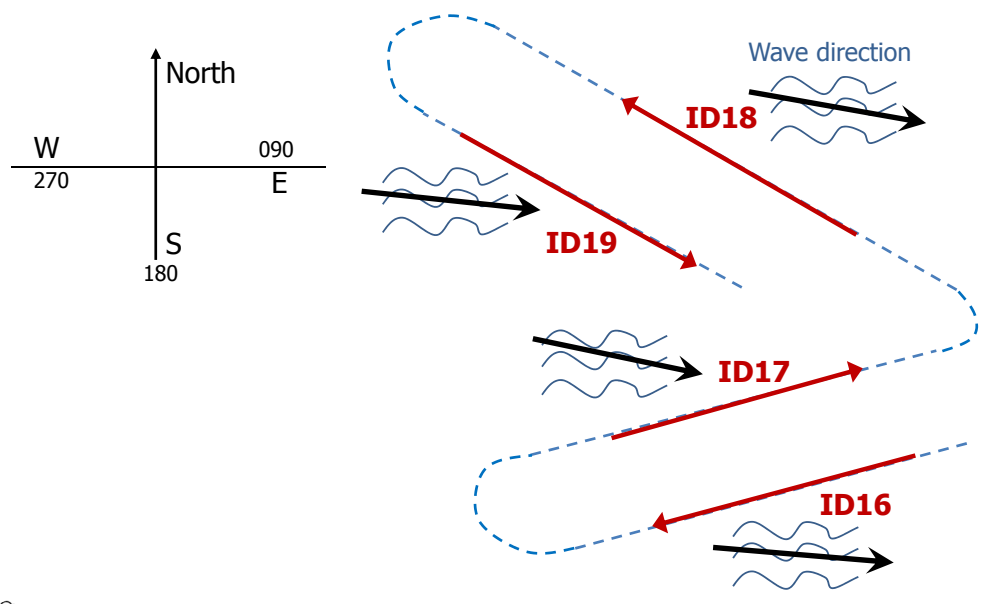

Figure 13: Geographic run pattern of the seakeeping tests during sea trials using R/V Gunnerus. 
Table 3: Sea trial conditions: Logged vessel speed $U$ and course relative to North, and estimated wave parameters by a free-floating wave buoy.

\begin{tabular}{cccccccc}
\hline & \multicolumn{2}{c}{ Vessel data } & & \multicolumn{4}{c}{ Wave parameters } \\
\cline { 2 - 3 } \cline { 5 - 7 } & $U[\mathrm{knots}]$ & Course & & $H_{s}[\mathrm{~m}]$ & $T_{z}[\mathrm{~s}]$ & $T_{p}[\mathrm{~s}]$ & $\vartheta_{\text {mean }}[\mathrm{deg}]$. \\
\hline ID16 & 10.4 & 255 & & 1.9 & 8.2 & 14.7 & 274 \\
ID17 & 10.4 & 076 & & 1.9 & 8.1 & 14.2 & 282 \\
ID18 & 10.4 & 300 & & 1.8 & 7.8 & 13.9 & 275 \\
ID19 & 10.3 & 120 & & 2.1 & 8.2 & 14.0 & 279 \\
\hline
\end{tabular}

at each of the run paths shown in Figure 13. Sometimes, the mean wave direction may be decomposed into swell and wind sea directions but in the studied test cases the swell component contains the vast parts of the total energy. This can be seen easily from plots of the frequency-wise energy distributions represented by the estimated wave spectra shown in Figure 14. The individual plots in the figure contain both the estimate of the ship motion-based result and that of the free-floating wave buoy. Generally, it is observed that the agreement between the matching sets of results - ship motion-based vs. wave buoy - is fairly good. The integrated wave parameters of the brute-force spectral
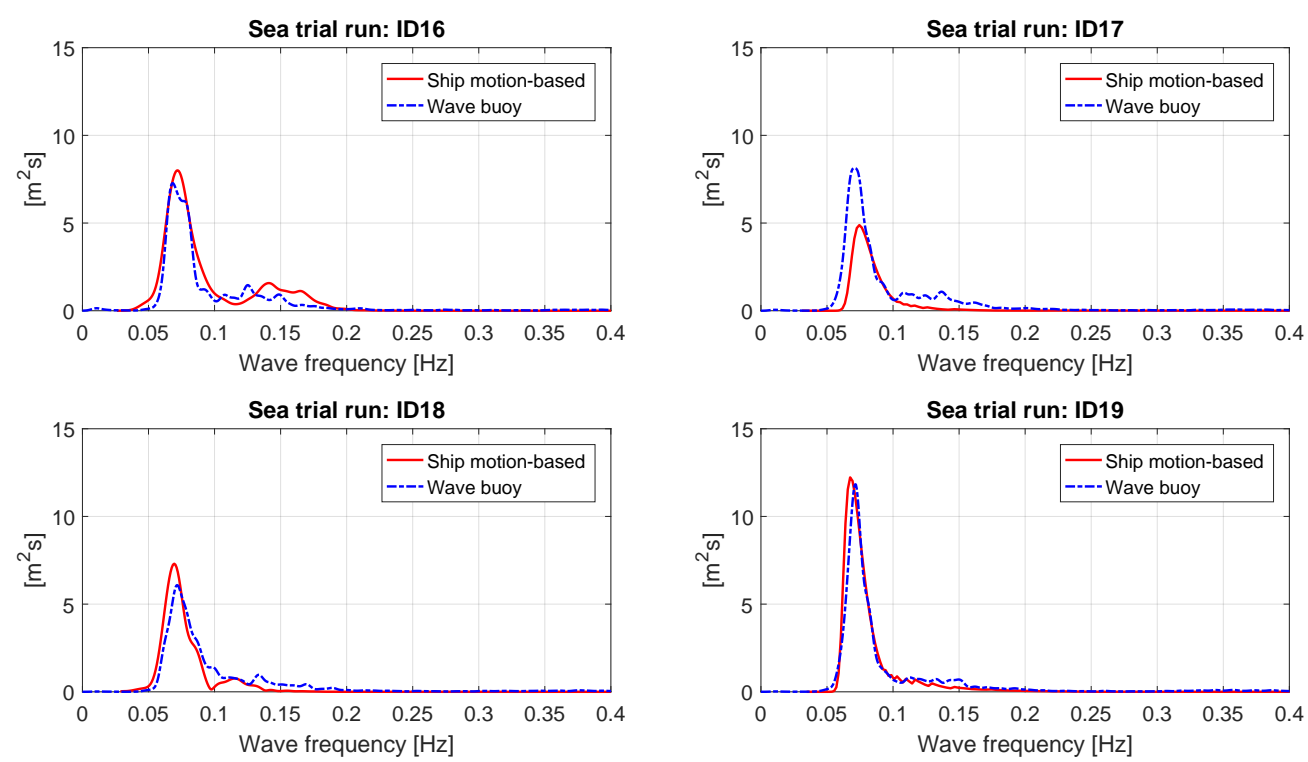

Figure 14: Frequency-wise energy spectra estimated using wave-induced vessel motions obtained from sea trials data; the estimates by a free-floating wave buoy are included as well. 
Table 4: Estimated wave parameters obtained by the brute-force spectral approach applied to full-scale sea trial data. The numbers in parentheses yield the corresponding number of the free-floating wave buoy.

\begin{tabular}{ccccc}
\hline & $H_{s}[\mathrm{~m}]$ & $T_{z}[\mathrm{~s}]$ & $T_{p}[\mathrm{~s}]$ & $\vartheta_{\text {mean }}$ \\
\hline ID16 & $2.1(1.9)$ & $9.9(8.2)$ & $13.9(14.7)$ & $280(274)$ \\
ID17 & $1.3(1.9)$ & $11.0(8.1)$ & $13.4(14.2)$ & $310(282)$ \\
ID18 & $1.7(1.8)$ & $12.6(7.8)$ & $14.4(13.9)$ & $265(275)$ \\
ID19 & $2.0(2.1)$ & $11.9(8.2)$ & $14.8(14.0)$ & $245(279)$ \\
\hline
\end{tabular}

approach are shown in Table 4, which includes also the results of the free-floating wave buoy in parentheses. It is noteworthy that in the brute-force spectral approach the mean wave direction can be calculated from the estimated relative wave heading ( $\chi$ which is the outcome of the algorithm) and the specific vessel course. As a general remark on the (comparisons of) sea state estimates, it must be remembered that it is impossible to obtain the very truth, since, in reality, smaller variations in wave conditions affect the results in location and time. As such, it may in this case not necessarily be the results of the free-floating buoy that should be considered as the 'base metric', claiming the wave buoy to be the more correct one, since the physical distance between the wave buoy and the individual run path in principle excludes any direct comparison.

For more detailed analyses of the full-scale sea trial data, reference is given to future work. For instance, it should be interesting to look closer on the influence, if any, of the exact value of forward-speed. The given speeds in Table 3 are the logged speed-throughwater, with the engine delivering constant and identical power $(2 \times 250 \mathrm{~kW})$ in all runs. However, during the tests there was a clear sign of sea current in the testing area, and an actual measurement indicated a strength of about 1 knots towards East-North East. It is obvious that motion transfer functions must be calculated with respect to speedthrough-water, but for the estimation algorithm itself it is not too obvious, since it is the relative speed between ship and waves that governs the mapping of encounter to absolute (wave) frequencies. 


\section{Conclusions and further work}

It has been shown that the presented brute-force estimation procedure performs well and makes accurate prediction of the on-site sea state; this goes for the integrated wave parameters but also for the more detailed frequency-wise distribution of wave energy, including the mean wave direction. Tests were made on both artificially generated data and on full-scale data from sea trials, and good estimating results were obtained in both cases. In this respect, the brute-force wave estimation procedure has a performance comparable to many of the other shipboard estimation techniques relying on the wave buoy analogy. However, the computational efficiency of the present procedure is significantly improved with estimation speed in the order of a few seconds in contrast to minutes for the other well-tested estimation procedures based on Bayesian modelling or parametric optimisation. On the first hand, this makes the present procedure useful for realtime onboard control and decision support tools focused on (autonomous) marine operations, where computational efficiency is vital. Secondly, the high computational speed means that it will be possible to integrate, in realtime, probabilistic calculations directly in the sea state estimation computations; something that cannot be made with other estimation techniques as they require too long computational time for the single spectrum estimates.

In the future, important and suggested work on the presented estimation procedure may be considering points on the following list, which by no means is exhaustive and does not necessarily include (sub)work already mentioned in the main text. Thus, further work should

- apply the estimation algorithm to (new) experimental data, including model-scale and full-scale, where motion measurements obtained on various types of ships (without and with advance speed) are analysed;

- conduct sensitivity studies to examine, for instance, the influence of spectrum discretisation used in the cross spectral analysis, the influence of the value of forwardspeed, the influence that the use of different motion, or response, combinations has on the final spectrum estimate, depending on the operational and environmental conditions; as all this knowledge will be essential for real-case application of the algorithm; 
- optimise the estimation procedure for computational speed;

- extend the brute-force approach to work for wave systems composed by swell and wind sea (subsystems) from different directions.

\section{Acknowledgement}

The first author would like to acknowledge his long-time colleague and friend Professor Emeritus Jørgen Juncher Jensen. It is interesting that Jørgen many years ago initiated a study introducing an (unpublished) idea for sea state estimation somewhat in line with the brute-force solution presented in this article.

This work was supported by the Research Council of Norway through the Centres of Excellence funding scheme, project number 223254 NTNU AMOS. The set of sea trials data was made available by the SIMVAL KPN project, financed through grant number 225141/O70 by Rolls-Royce Marine and the Norwegian Research Council. Thanks to Rolls-Royce Marine for permission to publish the results.

\section{References}

[1] M. Ludvigsen, A. Sørensen, Towards integrated autonomous underwater operations for ocean mapping and monitoring, Annual Reviews in Control 42 (2016) 145-157.

[2] T. Perez, Ship Seakeeping Operability, Motion Control, and Autonomy - A Bayesian Perspective, IFAC-PapersOnLine 48-16 (2015) 217-222.

[3] R. Veal, M. Tsimplis, The integration of unmanned ships into the lex maritima, Lloyd's Maritime \& Commercial Law Quarterly (in press) .

[4] T. Iseki, K. Ohtsu, Bayesian estimation of directional wave spectra based on ship motions, Control Engineering Practice 8 (2000) 215-219.

[5] E. A. Tannuri, J. V. Sparano, A. N. Simos, J. J. D. Cruz, Estimating directional wave spectrum based on stationary ship motion measurements, Applied Ocean Research 25 (2003) 243-261.

[6] R. Pascoal, C. G. Soares, A. J. Sørensen, Ocean Wave Spectral Estimation Using Vessel Wave Frequency Motions, Journal of Offshore Mechanics and Arctic Engineering 129 (2007) 90-96.

[7] R. Pascoal, L. P. Perera, C. G. Soares, Estimation of Directional Sea Spectra from Ship Motions in Sea Trials, Ocean Engineering 132 (2017) 126-137.

[8] U. D. Nielsen, Estimations of on-site directional wave spectra from measured ship responses, Marine Structures 19 (2006) 33-69.

[9] U. D. Nielsen, Introducing two hyperparameters in Bayesian estimation of wave spectra, Probabilistic Engineering Mechanics 23 (2008) 84-94. 
[10] U. D. Nielsen, J. J. Jensen, P. T. Pedersen, Y. Ito, Onboard monitoring of fatigue damage rates in the hull girder, Marine Structures 24 (2011) 182-206.

[11] U. D. Nielsen, A concise account of techniques available for shipboard sea state estimation, Ocean Engineering 129 (2017) 352-362.

[12] A. H. Brodtkorb, U. D. Nielsen, A. J. Sørensen, Sea State Estimation Using Vessel Response in Dynamic Positioning, Applied Ocean Research 70 (2018) 76-86.

[13] R. Bhattacharyya, Dynamics of Marine Vehicles, John Wiley \& Sons, 1978.

[14] R. Beck, W. Cummins, J. Dalzell, P. Mandel, W. Webster, Vol. III: Motions in Waves and Controllability, in: E. Lewis (Ed.), Principles of Naval Architecture, Second Revision, SNAME, 1-188, 1989.

[15] J. Journée, W. Massie, Offshore Hydromechanics, lecture notes in course offered at TU Delft, 2001

[16] U. D. Nielsen, Transformation of a wave energy spectrum from encounter to absolute domain when observing from an advancing ship, Applied Ocean Research 69 (2017) 160-172.

[17] R. Pascoal, C. G. Soares, Non-parametric wave spectral estimation using vessel motions, Applied Ocean Research 30 (2008) 46-53.

[18] U. D. Nielsen, The wave buoy analogy - estimating high-frequency wave excitations, Applied Ocean Research 30 (2008) 100-106.

[19] N. Montazeri, U. D. Nielsen, J. J. Jensen, Estimation of wind sea and swell using shipboard measurements - A refined parametric modelling approach, Applied Ocean Research 54 (2016) 73-86.

[20] U. D. Nielsen, D. C. Stredulinksy, Sea state estimation from an advancing ship - A comparative study using sea trial data, Applied Ocean Research 34 (2012) 33-44.

[21] U. D. Nielsen, Z. Lajic, J. J. Jensen, Towards fault-tolerant decision support systems for ship operator guidance, Reliability Engineering and System Safety 104 (2012) 1-14.

[22] J. J. Jensen, A. E. Mansour, A. S. Olsen, Estimation of ship motions using closed-form expressions, Ocean Engineering 31 (2004) 61-85.

[23] A. Mansour, J. Jensen, A. Olsen, Fast Evaluation of the Reliablity of Container Securing Arrangements, in: Proceedings of PRADS'04, Travemünde, Germany, 577-585, 2004.

[24] J. J. Jensen, J. Capul, Extreme response predictions for jack-up units in second order stochastic waves by FORM, Probabilistic Engineering Mechanics 21 (2006) 330-338.

[25] J. Mas-Soler, A. N. Simos, E. A. Tannuri, Estimating on-site wave spectra from the motions of a semi-submersible platform: An assessment based on model scale results, Ocean Engineering (under review) 153 (2018) 154-172.

[26] T. Iseki, An improved stochastic modeling for bayesian wave estimation, in: Proc. of OMAE 2012, ASME, Rio de Janeiro, Brazil, 2012.

[27] I. M. V. Andersen, G. Storhaug, Dynamic Selection of Ship Responses for Estimation of on-site Directional Wave Spectra, in: Proc. 31st OMAE, Rio de Janeiro, Brazil, 2012.

[28] S. Steen, Ø. Selvik, V. Hassani, Experience with rim-driven azimuthing thrusters on the research ship Gunnerus, in: Proc. of High-Performance Marine Vessels, Cortona, Italy, 2016.

[29] Sintef Ocean, ShipX, http://www.sintef.no/programvare/shipx/, Accessed: 2017-08-23. 
[30] Datawell BV, Directional Waverider, http://www.datawell.nl/, Accessed: 2017-08-23. 


\section{Appendix A. Initial wave heading estimation}

In order to illustrate the initial heading estimation Figure A.15 is considered. The two plots in the figure are the (initial brute-force) outcomes of the estimation procedure when it has been applied to artificially generated motion data; the details about the vessel in study, about the time history generation, etc., have been presented in Section 5. In the given case (Fig. A.15), two different sets of time history recordings of \{heave,roll,pitch\} are used as input to the estimation procedure. The two sets of motion recordings have been obtained/simulated using a short-crested wave system derived from the same type of wave spectrum; herein taken as a Bretschneider spectrum (see Sec. 5) with $H_{s}=2.0 \mathrm{~m}$ and $T_{m}=6.5 \mathrm{~s}$. The only difference between the two sets of motion measurements is that the one set applies for a mean wave heading $\chi=60 \mathrm{deg}$. (left-side plot) and the other set for $\chi=120 \mathrm{deg}$. (right-side plot). In accordance with the selection process described in Subsection 3.2.1, it is found that the smallest variation between the estimated $H_{s^{-}}$ values is found for $\hat{\chi}=55 \mathrm{deg}$. and $\hat{\chi}=125 \mathrm{deg}$. for the left- and right-hand side plots, respectively. At these particular headings, the average significant wave heights are $\hat{H}_{s}=2.2 \mathrm{~m}(\hat{\chi}=55 \mathrm{deg}$.$) and \hat{H}_{s}=1.7 \mathrm{~m}(\hat{\chi}=125 \mathrm{deg}$.$) , as calculated from the two$ corresponding optimum wave spectrum estimates, cf. Eq. (15).
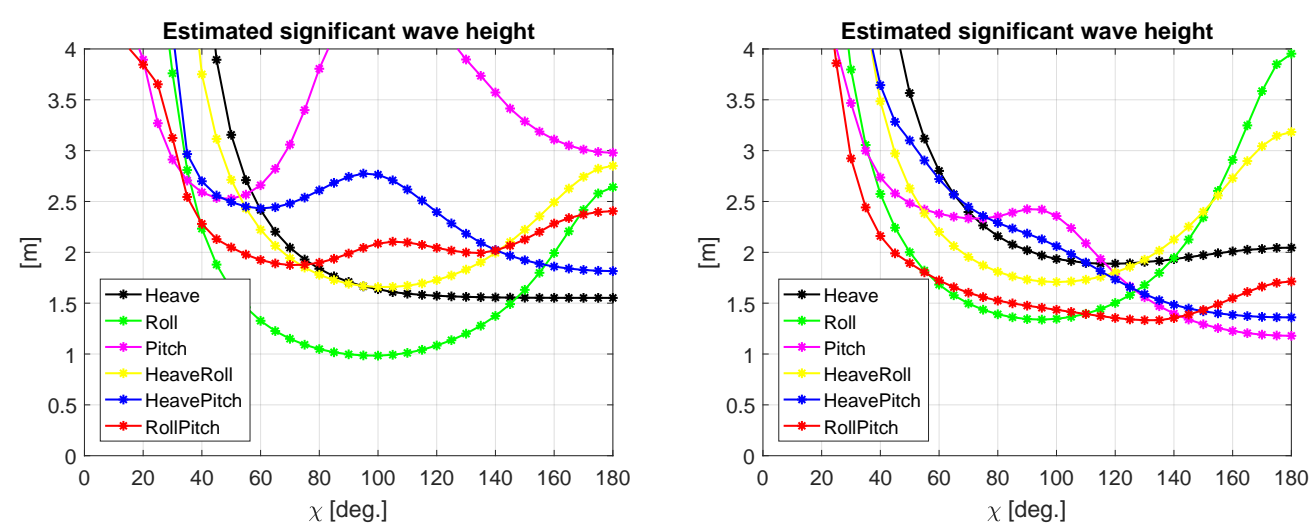

Figure A.15: Variation of significant wave height $H_{s}$ with mean relative wave heading $\chi$ using different motion components measured in a short-crested wave system. Generating parameters are for the left-side plot $\chi=60 \mathrm{deg}$. and $H_{s}=2.0 \mathrm{~m}$, respectively, and for the right-side plot $\chi=120 \mathrm{deg}$. and $H_{s}=2.0 \mathrm{~m}$. 


\section{Appendix B. Wave spectrum and motion transfer function of simulation study}
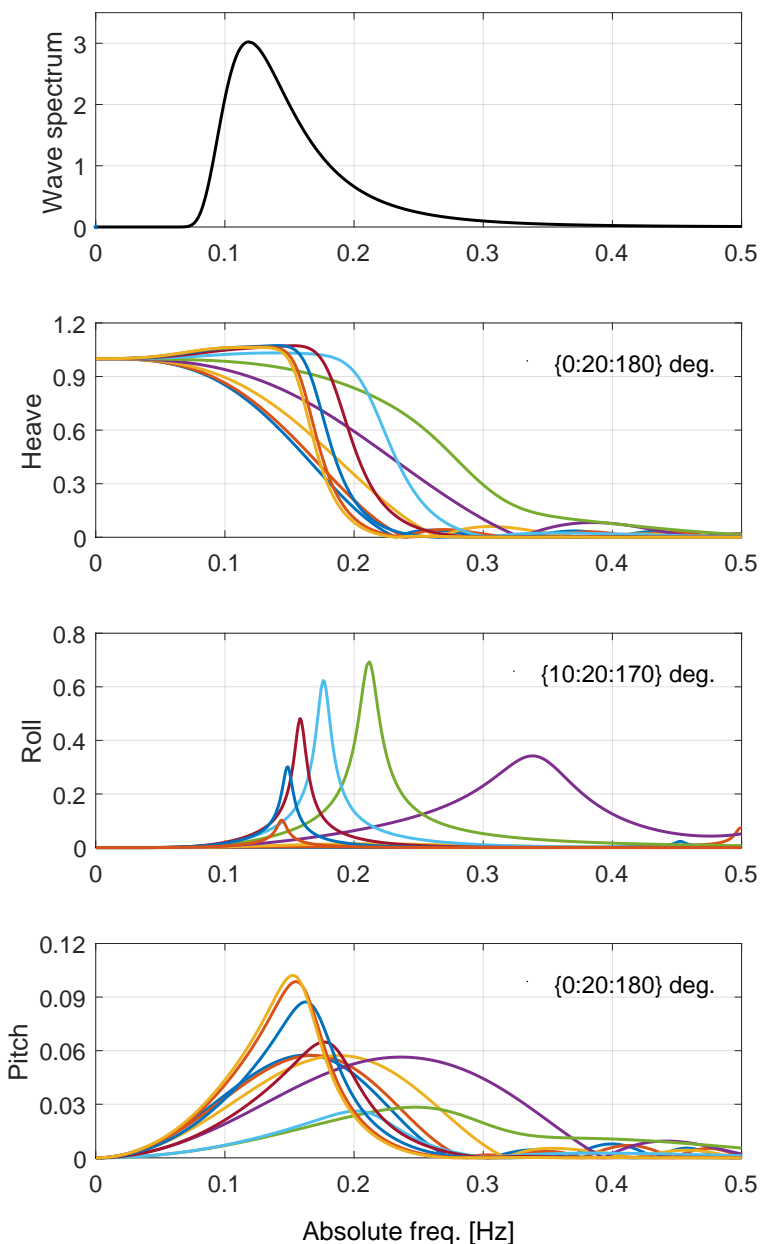

Figure B.16: A Bretschneider wave spectrum (uppermost plot) with $T_{m}=6.5 \mathrm{~s}$ and $H_{s}=2.0 \mathrm{~m}$ is used as the generating spectrum for the time series simulations of $\{$ heave, roll, pitch $\}$ at forward speed $U=10$ knots. The modulus (amplitudes) of the motion transfer functions are shown below the wave spectrum, and the results for different relative headings are presented, leaving the detailed legends out since the interest is merely the 'qualitative variation' between the different headings. Units: Wave spectrum $\left[\mathrm{m}^{2} \mathrm{~s}\right] ;$ Heave $[\mathrm{m} / \mathrm{m}] ;$ Roll $[\mathrm{rad} / \mathrm{m}] ;$ Pitch $[\mathrm{rad} / \mathrm{m}]$. 


\section{Appendix C. Time series simulation of wave-induced responses}

The wave elevation and the corresponding vessel motions are considered as Gaussian distributed. Hence, in linear, short-crested waves the time history record $R(t)$ of a waveinduced motion component can be generated using a set of uncorrelated, standard normal distributed variables $u_{n m}$ and $\bar{u}_{n m}$ [e.g. 24],

$$
R(t)=\sum_{n=1}^{N} \sum_{m=1}^{M}\left[u_{n m} c_{n m}(t)+\bar{u}_{n m} \bar{c}_{n m}(t)\right]
$$

The deterministic coefficients $c_{n m}(t)$ and $\bar{c}_{n m}(t)$ are for an advancing vessel given by,

$$
\begin{aligned}
c_{n m}(t) & =\sigma_{n m}\left|\Phi_{R}\left(\omega_{0, n}, \mu_{m}+\chi\right)\right| \cos \left(\omega_{e, n m} t+\epsilon_{R}\left(\omega_{0, n}, \mu_{m}+\chi\right)\right) \\
\bar{c}_{n m}(t) & =-\sigma_{n m}\left|\Phi_{R}\left(\omega_{0, n}, \mu_{m}+\chi\right)\right| \sin \left(\omega_{e, n m} t+\epsilon_{R}\left(\omega_{0, n}, \mu_{m}+\chi\right)\right) \\
\sigma_{n m}^{2} & =\hat{S}\left(\omega_{0, n}, \mu_{m}\right) \Delta \omega_{0, n} \Delta \mu_{m}
\end{aligned}
$$

where the modulus (amplitude) and the phase of the motion transfer function are $\left|\Phi_{R}\left(\omega_{0, n}, \mu_{m}+\chi\right)\right|$ and $\epsilon_{R}\left(\omega_{0, n}, \mu_{m}+\chi\right)$, respectively, for the particular motion component $R$. The generating wave energy spectrum $\hat{S}\left(\omega_{0}, \mu\right)$ is discretised at $N$ frequencies and $M$ directions. The present formulation considers time histories of wave-induced motion components observed from the advancing vessel. This means that the encounter frequency $\omega_{e}$, appearing in the deterministic coefficients, is given by, cf. Eq. (1)

$$
\omega_{e}=\left|\omega_{0}-\omega_{0}^{2} \psi\right|, \psi=\frac{U}{g} \cos \chi
$$

for any absolute frequency $\omega_{0}$. 